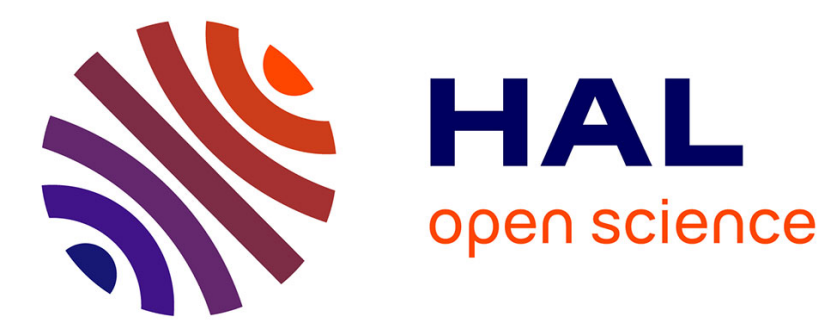

\title{
SPH modeling of fluid-solid interaction for dynamic failure analysis of fluid-filled thin shells
} Fabien Caleyron, Alain Combescure, Vincent Faucher, S. Potapov

\section{To cite this version:}

Fabien Caleyron, Alain Combescure, Vincent Faucher, S. Potapov. SPH modeling of fluid-solid interaction for dynamic failure analysis of fluid-filled thin shells. Journal of Fluids and Structures, 2013, 39, pp.126-153. hal-00819622

\section{HAL Id: hal-00819622 https://hal.science/hal-00819622}

Submitted on 2 May 2013

HAL is a multi-disciplinary open access archive for the deposit and dissemination of scientific research documents, whether they are published or not. The documents may come from teaching and research institutions in France or abroad, or from public or private research centers.
L'archive ouverte pluridisciplinaire HAL, est destinée au dépôt et à la diffusion de documents scientifiques de niveau recherche, publiés ou non, émanant des établissements d'enseignement et de recherche français ou étrangers, des laboratoires publics ou privés. 


\title{
SPH modeling of fluid-solid interaction for dynamic failure analysis of fluid-filled thin shells
}

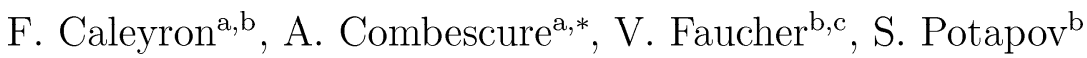 \\ ${ }^{a}$ Université de Lyon, CNRS, INSA-Lyon, LaMCoS UMR 5259, F-69621, France \\ ${ }^{b}$ LaMSID UMR EDF-CNRS-CEA 2832 \\ ${ }^{c}$ CEA DEN, DM2S, Laboratoire d'Etude de Dynamique, F-91191 Gif-sur-Yvette, France
}

\begin{abstract}
This work concerns the prediction of failure of a fluid-filled tank under impact loading, including the resulting fluid leakage. A water-filled steel cylinder associated with a piston is impacted by a mass falling at a prescribed velocity. The cylinder is closed at its base by an aluminum plate whose characteristics are allowed to vary. The impact on the piston creates a pressure wave in the fluid which is responsible for the deformation of the plate and, possibly, the propagation of cracks. The structural part of the problem is modeled using Mindlin-Reissner Finite Elements (FE) and Smoothed Particles Hydrodynamics (SPH) shells. The modeling of the fluid is also based on an SPH formulation. The problem involves significant Fluid-Structure Interactions (FSI) which are handled through a master-slave-based method and the pinballs method. Numerical results are compared to experimental data.
\end{abstract}

Keywords: SPH, fluid-structure interaction, impact, failure

*. Corresponding author : Tel : +33 4724364 26, Fax : +33 478890980

Email address: alain.combescure@insa-lyon.fr (A. Combescure) 


\section{Introduction}

The recent increase in terrorist threats has led to an intensification of research efforts in order to protect citizens and industrial installations. Numerous scenarios, such as the fall of a commercial aircraft on a civil engineering structure, must be envisaged.

In this context, the analysis of the fracture and leakage of a thin fluidfilled structure, such as an aircraft's fuel tank, is an especially important undertaking. This is a complex problem which involves highly nonlinear phenomena both in the fluid (sloshing, splashing, shock waves, cavitation) and in the structure (large strains, damage, cracking, dynamic fracture). Moreover, the interface between the two media can undergo drastic changes during the process, for example in the case of significant movements of the free surface of the fluid or the rupture of the tank.

The simulation of this type of problem is a real scientific challenge for which research is still ongoing. The literature recounts different approaches which are briefly summarized below.

Some authors, such as Timm in (Timm, 2003), use the classical Lagrangian formulation of the FEM. However, this approach has shortcomings : in particular, highly distorted meshes lead to numerical errors and a decrease in time steps which affect the effectiveness and efficiency of the method. These problems can sometimes be circumvented through erosion of the distorted elements, but this approach precludes the conservation of the mass and energy of the model throughout the calculation, except if the eroded elements are replaced by debris as in (Sauer, 2010). 
Sometimes, Eulerian approaches are also used, e.g. in (Anderson et al., 1999), especially to study the fluid part of the problem. Indeed, in this case, the mesh does not vary with time and, thus, it remains valid regardless of the strains undergone by the material. However, in this context, the study of the structure is difficult, particularly if it is thin and ruptures. Therefore, the Eulerian fluid model is sometimes coupled with a FE model of the structure.

An intermediate approach consists in using an Arbitrary LagrangianEulerian (ALE) model, which enables Lagrangian calculations to be carried out while leaving the possibility for the mesh to evolve independently of the medium in order to avoid, for example, excessive distortions. The ALE model can be used used to represent the whole problem or the fluid alone (Varas et al., 2009b) while the fluid tank and the impactor are modeled using the FEM.

Finally, an interesting approach for this class of problems consists in using meshless methods. Indeed, these methods enable large strains and the introduction of discontinuities such as cracks to be handled naturally. One can cite the works of Maurel et al. (Maurel et al., 2009; Potapov et al., 2009) and Caleyron et al. (Caleyron et al., 2011), who proposed a model based entirely on the SPH method for the analysis of tanks under impact. Rabczuk et al. also proposed models based on the Element-Free Galerkin (EFG) meshless method for the resolution of this type of problem (Rabczuk et al., $2007,2009)$. In some cases, the meshless model is used only for the fluid part of the problem (Varas et al., 2009b), sometimes even in an adaptive way as a function of the progression of the projectile into the fluid (Sauer, 2010). In this paper, an SPH model is used for the fluid for the reasons mentio- 
ned previously. The tank is modeled classically using the FEM, except in the zones subjected to fracture. In this case, a specific SPH shell model presented in (Maurel et al., 2009; Potapov et al., 2009; Caleyron et al., 2011) is used locally.

The availability of reliable experimental data is also important in order to evaluate the performance of the numerical models thus defined. The experiments developed for that purpose must be simple, well-designed and properly instrumented as well as representative of the phenomenon being studied.

The literature abounds with experimental studies concerning the "hydrodynamic ram" (HRAM) phenomenon which occurs during high-speed impact on a fluid-filled tank (Disimile et al., 2009; Varas et al., 2009a; Freitas et al., 1996, 1997). In this case, the projectile transfers its kinetic energy to the structure via the fluid, which, thus, can lead to the complete failure of the tank. However, most of the studies focus mainly on the behavior of the fluid (shock waves, cavitation) while the strains in the structure remain small outside of the perforation zone.

Far fewer experimental works focus precisely on the fracture of the structure. One can cite the works by Timm (Timm, 2003) on the impact of a bullet onto an empty or water-filled cylinder for various impact velocities and cylinder dimensions. The author showed that for sufficiently high incoming velocities the impact results in the propagation of a longitudinal crack on the impacted side of the tank. Rabczuk et al. compared these experimental data with results from simulations using meshless models (Rabczuk et al., 2007, 2009). In (Sauer, 2010), Sauer studied the fragmentation of a 
cylinder impacted by a projectile. The cylinder was filled with water and, in some of the cases, presented precracks. The experimental data (residual velocity of the projectile, fracture mode, distribution of the fragments) were also compared to the results of simulations using SPH and FE models.

One can also mention interesting experiments involving the fracture of tubes (Chao and Shepherd, 2005) or tanks (Keesecker et al., 2003) under internal pressure. The corresponding simulations have been reported, for example, in (Rabczuk et al., 2007, 2009).

In this context, this paper presents an original experiment which is representative of a tank subjected to an impact. This enables us to study the behavior of a plate interacting with a fluid while controlling the phenomena involved : simple water flow, large strains, cracking or even the complete rupture of the tank. Some of the experimental data were already presented in (Potapov et al., 2009). This paper completes the previous publication by providing the full experimental results as well as the corresponding simulations.

The paper is divided into four sections. The first section presents the experimental setup. In the second section, the results are examined in detail and interpreted in order to emphasize the phenomena involved in the experiment. The third section is devoted to the description of the associated numerical model. In the last section, the results given by the model are compared to the experimental data. 


\section{Presentation of the tests}

The objective of the test campaign was to provide an experimental setup which would be simple, yet representative of an impact on a fluid-filled tank. Thus, the principle of the experiment was simple : a cylinder, closed by a metal plate which constituted the zone of interest, was filled with water, then fitted with a piston which was impacted by a mass. The impact created a pressure shock in the water, leading to a deformation of the specimen followed by the propagation of a crack and, ultimately, the leakage of the water contained in the piston. The experimental campaign was carried out at ONERA in Lille by J. Fabis in 2006. This section describes the experimental setup, the characteristics of the specimens used, the scenarios of the tests and the measurement system.

\subsection{The experimental setup}

The experimental setup is shown in Figure 1. It consisted essentially of a thick cylinder $\left(e_{c y l}=15 \mathrm{~mm}, D_{c y l}=80 \mathrm{~mm}\right)$ associated with a piston. Both elements were made of steel (APX Z15 CN 17.03). The cylinder was closed at the bottom by a plate which constituted the zone of interest of the experiment and whose characteristics were allowed to vary depending on the tests : different thicknesses, presence of a hole or precracks in order to observe different types of behavior. These specimens were made of aluminum (AU4G 2024 T351) so they could deform more easily than the cylinder. The cylinder was filled with water to form a column of height $H=\left(205-e_{p}\right) m m, e_{p}$ being the thickness of the specimen. The pressure shock in the water was created by the fall of a mass $M=245 \mathrm{~kg}$ onto the piston. In order to do that, we 
used the drop tower at ONERA in Lille and attached the mass to a carriage guided by a system of rails (see Figure 2). Two impact velocities, $V_{1}=2 \mathrm{~m} / \mathrm{s}$ and $V_{2}=5 \mathrm{~m} / \mathrm{s}$, were considered. These were obtained by selecting the proper carriage release height $H_{l}$ using the formula $H_{l}=V^{2} / 2 g$. The drop tower was installed on an $80 t$ foundation which enabled the crash zone to be uncoupled from the rest of the structure. The experimental setup allowed good monitoring of the experimental parameters : mass, impact velocity, angle of incidence, resonance frequencies, etc. Moreover, special care was given to the setting of the boundary conditions of the aluminum specimen, as explained in the following section.

Note : aluminum grade AU4G 2024 T351 was chosen because its behavior is hardly affected by the strain rate, thus enabling a simple constitutive relation to be used in the simulations.

\subsection{Characteristics of the specimens}

The aluminum specimens had various characteristics which enabled us to control the phenomena involved in the experiment (water flow, deformation of the bottom, cracking or fracture). The following configurations were tested :

- a circular hole with a diameter $d=14 \mathrm{~mm}$ or $d=20 \mathrm{~mm}$;

- a U-shaped precrack of length $3 \times 20 \mathrm{~mm}$;

- two $30 \mathrm{~mm}$ precracks forming an $\mathrm{X}$;

- no hole or precrack;

- a circular hole with a diameter $d=14 \mathrm{~mm}$ and two diametrically opposed $3 \mathrm{~mm}$ precracks ; 
- an I-shaped precrack of length $20 \mathrm{~mm}$.

The thickness of the part of the specimen in contact with the fluid was also allowed to vary. The plates were manufactured from a $200 \times 200 \times 25 \mathrm{~mm}$ aluminum block whose center part, of diameter $D_{c y l}=80 \mathrm{~mm}$, was spot-faced in order to obtain three different thicknesses : $e_{p}=25 \mathrm{~mm}$ (no spot-facing, thick specimen), $e_{p}=2 \mathrm{~mm}$ (thin specimen) and $e_{p}=1 \mathrm{~mm}$ (ultra-thin specimen). The different tests are listed in Table 4 along with the characteristics of the corresponding specimen. Examples of specimens in their initial state are shown in Figure 3.

As can be seen in Figure 3, the specimens were fitted with 12 circularly arranged holes to provide the connection with the cylinder. Additionally, they were fastened to the visualization space by means of 8 additional screws. Figure 4(a) represents a specimen attached to the experimental device according to the principle shown in Figure 4(b).

\subsection{The testing procedure}

The exact same procedure was followed for all the tests in order to achieve maximum reproducibility :

- the specimen was fastened to the end of the cylinder;

- the holes were sealed with adhesive tape;

- the cylinder was filled with water with no residual bubbles;

- contact between the carriage and the piston was established and the height measurement was set to zero; 
- the displacement sensor was verified;

- the carriage was raised to the desired release height;

- the spotlights were turned on behind a black screen to avoid overheating the setup;

- the measuring bridge automatic balance was performed, the pressure measurement reset to zero and the acquisition device and high-speed camera were wound up;

- the black screen was removed, the crash area cleared and the release activated.

\subsection{The acquisition apparatus}

A data acquisition chain was used to collect a variety of information precisely throughout the experiments :

- an optical displacement sensor was used to track the course of the carriage ;

- a high-extension strain gage $(\sim 15 \%)$ was affixed to each specimen in a zone which would be solicited by the pressure shock. These gages were associated with a $75 \mathrm{kHz}$ low-pass filter. The objective was to collect the strain state and the residual plasticity state at the position of the gage ;

- a piezoelectric pressure gage (measuring range 1,000 bar, eigenfrequency $150 \mathrm{kHz}$ ) was fastened to the underside of the cylinder in order to measure the evolution of the pressure at a point of the tank;

- a high-speed, high-definition camera (4,000 images per second and $768 \times 512$ pixel resolution) was used to monitor the behavior of the specimen and the flow. It also enabled us to estimate the velocity of 
the water outflow from the cylinder. High-power cold-light spotlights enabled the exposure time to be limited to $15 \mu \mathrm{s}$ without heating the specimens.

- a piezoelectric load sensor was placed under the specimen in the visualization space to measure the load exerted by the water jet;

- in order to acquire all these data synchronously, we used a multichannel analyzer equipped with acquisition cards capable of 1 million samples per second.

\section{Analysis of the tests}

This section presents the results obtained for the different configurations and discusses their interpretation. The tests which did not involve fracture (1 to $9,10,12$ and 13) will be studied first because they give an understanding of the behavior of the experimental setup. Then, the tests with fracture (11 and 14 to 19) will be analyzed.

\subsection{The tests without fracture}

\subsubsection{Observations}

Tests 1 through 9 (E14A-B-C2, E14A-B-C-D5, E20A2 and E20A5) were the simplest because the bottom was thick and had a single hole. Therefore, it deformed only slightly and the consequences of the impact reduced to the creation of a water jet spouting out of the cylinder. The curves plotted from the pressure measurements inside the cylinder for tests E14A-B-C2 and E14A-B-C-D5 are shown in Figures 5 and 6.

These signals can be divided into two parts : 
- Immediately following the impact, a pressure peak occurred and slightly affected the measurement. More precisely, this peak varied between 210 and 277 bars for tests E14A-B-C-D5, and between 58 and 83 bars for tests E14A-B-C2. This dispersion was probably due to the fact that the impact triggered a vibration of all the components of the device, which perturbed the measurements.

- After the impact, the flow through the hole became steady. Then, the results were highly reproducible, particularly for tests E14A-B-C-D5, which confirms the quality of the setup and of the measuring system. For all the tests, a highly reproducible pressure decrease associated with periodical variations was observed during the flow. Therefore, this decrease could not be due to measurement noise and must be attributed to a physical phenomenon, a conclusion made even stronger by the care with which the gages were chosen in order to eliminate measurement problems (150 kHz resonance frequency, $75 \mathrm{kHz}$ low-pass filter).

These periodic pressure variations for tests E14A-B-C-D5, E14A-B-C-D2, E20A2 and E20A5 were related to the observation of phenomena with the same frequency in the associated water jet (see Figure 8(a)). The water flow was highly turbulent with a Reynolds number of about 1. $e^{6}$. It started in the form of a "mushroom", as shown in Figure 7, when the water was expelled from the cylinder ; then, once the flow became steady, the jet came to a diameter slightly smaller than the hole and a bulb-shaped pattern was observed (see Figure 8(a)). These bulbs appeared during the flow at a constant frequency which was close to that observed for the pressure signal. At the end 
of the flow, the bulbs disappeared and a perfectly cylindrical jet could be observed briefly (see Figure $8(\mathrm{~d})$ ).

In the case of deformable specimens (such as in tests F14A2, FUA5 and FUA2), a periodical signal with the same frequency was also measured by the strain gage, as can be seen in the comparison of the pressure and strain signals for test F14A2, in which the gage was placed at a distance $\left(D_{\text {ext }}+d\right) / 4$ from the center of the plate (see Figure 9).

Finally, tests FUA2 and FUA5 presented no fracture or cracking either. Indeed, the U-shaped precrack flexed under pressure and opened to let the fluid escape. The opening angle of the tongue thus formed was a function of the impact velocity, as shown in Figure 10.

The water jet presented no bulbs because it was perturbed by the incompletely open tongue (see Figure 11).

However, the curves obtained from the pressure and strain gages had the same characteristics as in the previous tests. Indeed, Figure 12 shows the same in-phase periodical variations of the pressure and strain signals. (Here, the pressure gage was placed in the tongue's bending zone, as shown in Figure 3.) One can observe that in these two tests the strains reached significant levels (1.8\% for FUA2 and $2.0 \%$ for FUA5), which means that plasticity was taking place at the measurement point. This is confirmed by the existence of a residual opening of the tongues in Figure 10.

An analysis of the signals through Fourier series decomposition shows that the frequency observed was about $f \sim 1600 \mathrm{~Hz}$ for tests 1 through 9 , $f \sim 1000$ to $1200 \mathrm{~Hz}$ for test 10 and $f \sim 1300 \mathrm{~Hz}$ for tests 12 and 13 . 


\subsubsection{Interpretations}

Despite the apparent simplicity of tests 1 to 13 , the experimental data reveal the occurrence of complex phenomena. Indeed, the pressure and strain signals present in-phase periodical variations which can be compared to the occurrence, at the same frequency, of bulbs in the water stream. The frequency observed varied slightly with the geometry of the specimens (see Table 5). Higher frequencies were observed in the tests associated with a thick bottom.

The interpretation of these phenomena is not easy. The simplest explanation would consist in attributing the periodical variations to the shock wave traveling back and forth in the fluid column, but two arguments invalidate that explanation. First, according to that theory, the frequency observed should increase as the water level in the cylinder decreases, and not remain constant as was actually observed. Second, the measured frequency should be higher, of the order of $f=c /(2 H) \sim 4,000 \mathrm{~Hz}, c=1,450 \mathrm{~m} / \mathrm{s}$ being the velocity of sound in water.

In fact, the pattern shown in Figure 8(a) corresponds to an instability of the type which develops classically in highly turbulent jets, called PlateauRayleigh instability. For this instability to develop, the jet must first be solicited. The excitation frequency controls the frequency of occurrence of the patterns in the jet. In our case, it is likely that the shock solicited the eigenmodes of the fluid-structure oscillator formed by the experimental setup, leading to the development of bulb-shaped instabilities in the jet. Thus, the frequencies observed probably correspond to an eigenmode of the assembly. This is consistent with the decrease in frequency observed in the case of thin 
specimens. A simplified model of a cylinder filled with water was studied using the CASTEM software. The frequency of the first eigenmode of the model was found to be approximately $f \sim 1,300 \mathrm{~Hz}$, which confirms the previous reasoning. Figure 13 shows the associated deflection and the pressure isocurves in the fluid for $e_{p}=1 \mathrm{~mm}$.

\subsection{The tests with fracture}

Let us now consider tests 11 and 14 to 19. These tests involved crack propagations leading, in some cases, to the complete rupture of the specimen. These phenomena were combined to those described in the previous section. In tests TF14A5, F14A2-5 and TFA5, the rupture of the bottom at the level of the spot-facing circumference was observed. The final state of the specimens is shown in Figure 14. This phenomenon, which had not been anticipated at the time the specimens were designed, is due to the presence of stress concentrations, particularly shear stresses, at the level of the junction between the thin part and the thick bottom.

In the case of tests FXA2, FXA5, F14E3A5 and FE20A5, cracks propagated starting from the notches, as can be seen in Figure 15. However, contrary to the previous cases, one can note that the bottom was not completely ruptured.

\section{The numerical model}

This section presents the numerical model associated with the tests described previously. Since the correct eigenfrequency of the system appears to be important in order to achieve a good description of the problem, we chose 
to represent the various elements of the assembly explicitly. The model is shown in Figure 16.

\subsection{The structural model}

The cylinder was modeled using Q4GS thick shell finite elements (5 integration points through the thickness, selective integration) and the piston was represented as a material point connected to beam elements. The carriage was represented by its complete mass $M=245 \mathrm{~kg}$ concentrated into a material point. Depending on the case, the specimen was represented in two different ways :

- In the absence of crack propagation, Q4GS finite elements were used.

- In the presence of cracks, a shell model based on the SPH meshless method was used. Indeed, this model, which was presented in (Maurel and Combescure, 2008; Caleyron et al., 2011), is dedicated to fracture and crack propagation phenomena. A brief description will be given in Section 5.4.1.

The constitutive relations used were simple because the behavior of AU4G and APX materials can be considered to be independent of the strain rate. Therefore, Von Mises' elastic-plastic laws were used. A description of the parameters is given in Figure 17 and Tables 1 and 2. In the case of tests with fracture, Von Mises' elastic-plastic behavior of AU4G aluminum was coupled with a Lemaitre and Chaboche damage law. This coupling will be described in Section 5.3.1.

Both the specimen and the lower part of the cylinder were built-in. We also chose to fix the upper part of the cylinder radially in order to represent the massive head of the piston (see Figure 4(b). 
Altogether, the structural model consisted of about 1,700 Q4GS elements.

\subsection{The fluid model}

The fluid was modeled using the SPH method. Let us briefly review the equations of the model and their discretization. The fluid was assumed to be :

- perfect, i.e. exempt from viscosity. Thus, the equilibrium equation is :

$$
\frac{\partial \vec{v}}{\partial t}=-\frac{1}{\rho} \nabla p
$$

where $\vec{v}$ is the velocity vector, $p$ the pressure, $\rho$ the density and $t$ the time.

- weakly compressible. Thus, the continuity equation is :

$$
\frac{\partial \rho}{\partial t}+\rho \nabla \cdot \vec{v}=0
$$

The term $\partial \rho / \partial \vec{x} \cdot \vec{v}$ (where $\vec{x}$ is the position vector) was ignored, meaning that the density variations were assumed to be orthogonal to the velocity vector $\vec{v}$. This assumption is valid for water as long as the velocity remains small compared to the velocity of sound $c$.

- acoustic. Thus, the state equation is :

$$
d p=c^{2} d \rho
$$

This law is valid for slightly compressible fluids such as water as long as the velocity in the fluid remains small compared to the velocity of sound $c$. 
The fluid model is based on a classical SPH formulation, whose characteristics are the following :

- The SPH kernel $W_{i j}$ is a B3 spline.

- The equilibrium equation is discretized as :

$$
\left(\frac{\partial \vec{v}}{\partial t}\right)_{i}=-\sum_{j} m_{j}\left(\frac{p_{i}}{\rho_{i}{ }^{2}}+\frac{p_{j}}{\rho_{j}{ }^{2}}\right) \vec{\nabla} W_{i j}
$$

where $i$ is the current particle and $j$ is one of its neighbors of mass $m_{j}$. This is a symmetrical equation, which satisfies Newton's third law of motion (see (Gray et al., 2001)).

- The continuity equation is discretized as :

$$
\left(\frac{\partial \rho}{\partial t}\right)_{i}=\rho_{i} \sum_{j} \frac{m_{j}}{\rho_{j}}\left(\vec{v}_{i}-\vec{v}_{j}\right) \vec{\nabla} W_{i j}
$$

This equation was chosen because it makes the density variation be zero when the fluid flow is uniform.

- Artificial linear and quadratic viscosity terms were used as suggested in (Monaghan and Gingold, 1983) to stabilize the calculations in the case of shocks.

- The equations were expressed in the current configuration according to an updated Lagrangian formulation.

The water column was discretized using about 112,000 SPH particles of radius $R_{s p h}=1.025 \mathrm{~mm}$. Let us note that the particles were rather coarse compared to the dimensions of the specimen's opening : for example, the discretization used respectively 7 and 10 SPH particles for the circular holes of diameters $d=14 \mathrm{~mm}$ and $d=20 \mathrm{~mm}$. Indeed, SPH calculations represent 
a high CPU cost and the available computer resources did not allow the use of a larger number of particles.

\subsection{Fluid-structure interactions}

Two methods of handling the interactions between the fluid and the solid parts of the model were tested :

- The pinball method, which was initially developed for solid contact mechanics (Belytschko and Neal, 1991) and implemented into the EUROPLEXUS code for both finite elements (Casadei, 1973) and SPH particles (Maurel et al., 2009). In this approach, finite elements are filled with spheres, called pinballs, and the contact constraints are applied to these spheres rather than to the elements themselves. The treatment of interpenetration reduces to a simple check of the distance between two spheres. If $d_{12}$ is the distance between the centers of the two pinballs and $R_{1}, R_{2}$ are their radii, interpenetration occurs if :

$$
d_{12}<R_{1}+R_{2}
$$

This procedure is symmetrical because both solids play the same role and no distinction between a master and a slave is needed. Once contact has been detected, the contact forces are calculated by enforcing the impenetrability of the two impacting bodies. Lagrange multipliers rather than penalty functions were used, which required the introduction of a user-adjustable parameter. The contact forces must be such that for a nonviscous fluid :

$$
\left(\vec{v}_{1}-\vec{v}_{2}\right) \cdot \vec{n}=0
$$


where $\vec{v}_{1}, \vec{v}_{2}$ are the velocities of the pinballs and $\vec{n}$ a suitable direction normal to the contact surface. Much of the effectiveness and performance of the pinball algorithm depends on the choice of the expressions of $\vec{v}_{1}, \vec{v}_{2}$ and $\vec{n}$. In the particular case of the interaction between an SPH fluid particle and a shell finite element, the normal $\vec{n}$ is chosen to be the normal to the shell pinballs. This method is intended primarily for problems in which sliding and friction are not crucial : for example, in the case of two sliding planar bodies, oscillations perpendicular to the interface (because of the large radius of a single pinball per element) create problems. Moreover, the accuracy of the method in the case of highly distorted or irregular elements, or elements with a small topological thickness (e.g. beams or shells), is questionable.

- A master-slave method, sometimes called the sliding line method (2D) or the sliding surface method (3D) (Hallquist et al., 1985). One of the bodies in contact is designated as the master and the other as the slave. Interpenetration is detected by checking each slave node against the surface around the nearest master node. In the case of penetration, suitable contact forces are applied to the bodies. These forces can be calculated using a penalty method or a Lagrange multiplier. The Lagrange multiplier approach was chosen for this work. The masterslave method has several drawbacks : the interpenetration check requires complex geometric calculations (and some pathological contacts can remain undetected); the definition of some contact surfaces by the user may require voluminous input data; the concept of master and slave entities is artificial and creates a lack of symmetry in the formu- 
lation which makes the results depend upon the choice made by the user. However, it is often preferable to define as the master the body which is likely to be penetrated by the slave.

The capabilities of the two approaches to handle the fluid-structure interactions of the model shown in Figure 16 were tested.

The pinball method was found to produce poor results for the sliding contact between the cylinder shells and the SPH fluid particles. Indeed, due to the radii of the spheres, the normals were not exactly perpendicular to what can be viewed as the common interface of the two bodies in contact. The resulting contact force components tangent to the interface, even though they were small, produced undesired friction-like spurious effects which impaired the free relative sliding of the two bodies. Therefore, the master-slave approach was preferred for modeling the contact between the cylinder shells and the SPH fluid particles. In this particular case, the choice of the master and slave bodies was straightforward because SPH particles can be only slaves penetrating the finite element shells.

Contact between the SPH fluid and the aluminium specimens was handled using either sliding surfaces for specimens modeled by finite elements (no crack propagation) or the pinball method for specimens modeled by $\mathrm{SPH}$ shells (crack propagation).

Contact between the SPH fluid and the piston, which involves a rigid body for which the master-slave method is inapplicable, was handled using the pinball method. 
Remark : in the case of the SPH shell model, cylindrical pinballs were substituted for spherical pinballs in order to take into account the topological thickness of the shell.

\section{Comparison of the numerical and experimental results}

Tests E20A2 and E20A5 were studied first because they are the simplest and are representative of the series of tests 1 to 10 . They were chosen because of the specimens' larger hole which minimized the effect of the coarse SPH fluid model. Tests FUA2 and FUA5 were also simulated because, in that case, the bottom was thin and the opening of the tongue at a precise angle enabled us to verify the validity of the model. Then, test TF14A5 was studied in order to test the capability of the model to predict fracture as a result of a pressure shock. Finally, the crack propagations observed during test FXA5 were simulated.

\subsection{Tests E20A2 and E20A5}

These tests were simulated using two different boundary conditions for the specimen, as shown in Figure 18. Indeed, one can either consider that only the part of the specimen in contact with water is significant (Calculation 1) or try to position the built-in boundary more realistically (Calculation 2). The comparison of the two calculations enabled us to assess the influence of the boundary conditions on the results.

The pressure curves obtained with the two calculations are shown in Figures 19 and 20 (zoom). One can note that the pressure peak and variations 
immediately following the impact were correctly estimated. This is a welcome conclusion because this information is important in order to predict the possible rupture of the structure. However, after $t=2 \mathrm{~ms}$, the flow became steady and both the calculated pressure and its frequency of variation appeared to be too high by a factor of about 2 for Calculation 2 . The error was even worse in the case of Calculation 1. A comparison of the results of the two calculations leads one to conclude that small variations in the model's boundary conditions can lead to significant variations in the pressure signal. This problem is typical of the weakly compressible model used, for which a small variation in volume can lead to a significant pressure variation through the use of a state equation of the type :

$$
\delta p=c^{2} \delta \rho
$$

where $p$ denotes the pressure, $c$ the sound velocity in water and $\rho$ the mass density. Indeed, one can observe that a small error in the estimation of the mass density (due, e.g., to geometrical inaccuracies in the model or to a weakly consistent divergence operator) leads to high pressure variations by means of the state equation 8 . The example of water yields $\delta p \sim 20 \times 10^{5} \delta \rho$ because $c=1450 \mathrm{~m} / \mathrm{s}$, i.e., for $\delta \rho=1 \mathrm{~kg} / \mathrm{m}^{3}, \delta p \sim 20$ bars. This aspect has been signaled in the literature on the hydrodynamic SPH method, for example in Reference (Lee et al., 2010). Some authors attempted to improve the estimation of the pressure by using Moving Least Square (MLS) functions (Colagrossi and Landrini, 2003), by applying filters to various quantities (Dalrymple and Rogers, 2006) or by using an incompressible approach (Lee et al., 2006).

Thus, our idealized model is likely to be a little too stiff, leading to an 
overestimation of the pressure. Moreover, the coarseness of the SPH fluid model probably contributes to underestimating the leakage flow, which also induces an overpressure in the model. These tendencies, as explained before, are further amplified by the use of a weakly compressible model. A mesh which would be both more accurate (including the visualization space, the fastening screws, or even the whole drop tower) and more refined (SPH fluid discretization) along with an improved fluid model would probably lead to better results.

The other quantities obtained in the simulation matched the experimental data correctly. Figure 21 shows the trajectory of the carriage. Figure 22 shows the outgoing velocity of the water jet, which was calculated at $97 \mathrm{~m} / \mathrm{s}$, close to the estimated $92 \mathrm{~m} / \mathrm{s}$ obtained from the film recorded by the highspeed camera. Finally, Figure 23(b) gives the shape of the jet at $t=3 \mathrm{~ms}$. One can observe the presence of bulbs which are comparable, although less pronounced, to those observed experimentally in Figure 8(a). The simulation showed that these bulbs are associated with local variations in the velocity field in the jet as a direct consequence of the periodical pressure variations in the cylinder.

The results of test E20A2 are similar and will be mentioned only briefly. Here, as in all subsequent simulations, only the boundary condition corresponding to Calculation 2 was used. The pressure curves, which are similar to the previous ones, are shown in Figure 26. The water outflow velocity obtained through the simulation was about $44 \mathrm{~m} / \mathrm{s}$, which is close to the experimental value of about $45 \mathrm{~m} / \mathrm{s}$, as shown in Figure 27 . 


\subsection{Tests FUA2 and FUA5}

Let us now present and analyze the results of tests FUA2 and FUA5. These calculations were similar to the previous ones because no cracking occurred. In these two cases, the specimen was thin $\left(e_{p}=2 \mathrm{~mm}\right.$ and included a $20 \mathrm{~mm}$-wide U-shaped notch. This formed a tongue which, under the applied pressure, flexed and opened to a precise angle which was a function of the impact velocity. The interesting aspect of this test was for us to verify whether the model is capable of predicting the correct opening angle, i.e. handling the FSIs around the notch correctly. As before, the bottom was meshed using Q4GS finite elements because there was no propagating crack. The notch was modeled simply by duplicating the nodes along the cutting line.

The pressure curve obtained by the calculation for test FUA5 is shown and compared to the experimental results in Figure 28 ; a zoom on the impact zone is shown in Figure 29. The conclusions are the same as for the previous tests : the pressure estimate is good immediately after the impact, then deteriorates once the flow becomes steady. After $t=2 \mathrm{~ms}$, one can see that the pressure is overestimated by a factor of 2 and that the periodical variations in the calculated signal have disappeared.

Figure 30 shows a global view of the model at $t=3.5 \mathrm{~ms}$ while Figure 31 compares the experimental and numerical deflections of the specimen. There is an inherent error in that the numerical deflection, which still contains elastic strains (because the calculation was stopped before all the water had been ejected), is compared to the experimental deflection, which contains only plastic strains. Nevertheless, it is legitimate to neglect these elastic strains 
554

because the total strains are very large (about $2 \%$ for test FUA5 and $1.8 \%$ for test FUA2). The calculated opening angle $\alpha$, equal to about $\alpha \sim 50^{\circ}$, is very close to the experimental value. This shows that the FSIs around the specimen were correctly taken into account, which is also confirmed by looking at the strains near the zone where the tongue flexed, for which Figure 32 shows similar experimental and numerical results.

The numerical results of test FUA2 were slightly less good, but similar. They will be presented only briefly. The pressure curves are given in Figures 33 and 34 (zoom) and present the same characteristics as before. The opening angles of the tongue are compared in Figure 35. One can note that the match is less good than for test FUA5 because the opening predicted by the model seems a little too wide. The explanation probably lies in the coarse mesh used for the SPH fluid : indeed, a rapid calculation shows that for $\alpha \sim 20^{\circ}$ the size of the opening is about $3 \mathrm{SPH}$ particles, which is very coarse. Therefore, one could expect better results by using a more refined SPH discretization.

\subsection{Test TF14A5}

Like tests F14A2-5 and TFA5, test TF14A5 presented a ruptured bottom along the spot-facing circumference. The final state of the specimens is given in Figure 14. This phenomenon, which had not been anticipated at the time the specimens were designed, is due to the presence of stress concentrations, particularly shear stresses, at the level of the junction between the thin part and the thick bottom. 


\subsubsection{The damage model}

The crack's path was known and simple, which enabled the fractured specimen to be modeled using the FEM. In order to do that, we meshed the bottom using Q4GS finite elements again, and Von Mises' plasticity theory was coupled with Lemaitre and Chaboche's damage theory (Lemaitre et al., 1985).

In this case, the damage originates in the shear strain, which induces plasticity, and in the volume strain, which induces the growth of cavities and cracks. By expressing that the damage is governed by the shear and volume strain energies (Lemaitre et al., 1985), one obtains the following damage criterion $f_{D}$ :

$$
f_{D}=\left[\frac{2}{3}(1+\nu)+3(1-2 \nu)\left(\frac{\sigma_{H}}{\sigma_{e q}}\right)^{2}\right] p-\epsilon_{p}^{s} \leq 0
$$

where $\sigma_{H}$ denotes the hydrostatic stress, $\sigma_{e q}$ Von Mises' equivalent stress, $\sigma_{H} / \sigma_{e q}$ the triaxiality rate of the stresses and $\epsilon_{p}^{s}$ the threshold equivalent plastic strain. This criterion is known as the elastic energy density recovery rate criterion. The evolution of the damage is given by Lemaitre and Chaboche's law (Lemaitre et al., 1985) :

$$
\dot{D}=\frac{D_{c}}{\epsilon_{p}^{c}-\epsilon_{p}^{s}}\left[\frac{2}{3}(1+\nu)+3(1-2 \nu)\left(\frac{\sigma_{H}}{\sigma_{e q}}\right)^{2}\right] \dot{p}
$$

Three material parameters play a role in the evolution of the damage :

- the threshold equivalent plastic strain $\epsilon_{p}^{s}$ beyond which damage occurs, i.e. cavities and microcracks begin to grow,

- the critical equivalent plastic strain $\epsilon_{p}^{c}$ beyond which fracture of the material occurs, 
- the critical damage $D_{c}$ reached when $\epsilon_{p}=\epsilon_{p}^{c}$. Physically, $D_{c}$ represents the volume fraction of the defects at the fracture point.

Classically, this damage model is affected by a mesh dependency problem. Indeed, simulations involving a material with a negative stress-strain slope fail to give satisfactory results in the sense that, close to fracture, strains and damage are often artificially localized within a single mesh element. Thus, the path of what one may call the crack depends on the position of the elements. Moreover, if one refines the mesh, the damaged zone remains limited to the width of an element and critical damage is reached more rapidly ( $D_{c}$ is reached after a constant number of time steps). Thus, the energy dissipated in the fracture and the time to fracture tend to zero for an infinitely refined mesh. This is not physically consistent because it would require an infinite growth rate of the microcracks and cavities.

It was proven in (Bazoant and Belytschko, 1985; Needleman, 1988) that the origin of the problem resides in the transformation of the initial hyperbolic problem into an ill-posed elliptic problem when the material softens. Then, the propagation velocity of the waves becomes complex. Thus, the first element of the mesh which assumes softening behavior fails to transmit strains to its neighbors, which creates the problems mentioned previously.

The solutions proposed by the community to alleviate this difficulty consist in preserving the hyperbolic character of the equations. Most of these solutions boil down to treating the damage in a nonlocal manner by introducing a characteristic length or a characteristic time which reflects the interaction of one entity of the mesh with the others. In this work, we chose to introduce a characteristic time by using a model with a delay effect (Ladeveze, 1991; 
Ladevèze, 1992).

Consequently, the previous damage law was modified as follows :

$$
\dot{D}_{n c}=\frac{D_{c}}{\epsilon_{p}^{c}-\epsilon_{p}^{s}}\left[\frac{2}{3}(1+\nu)+3(1-2 \nu)\left(\frac{\sigma_{H}}{\sigma_{e q}}\right)^{2}\right] \dot{p}
$$

$\dot{D}_{n c}$ being what we call the uncorrected damage. Then the damage rate is given by (Allix and Deu, 1997) :

$$
\dot{D}=\frac{1}{\tau_{c}}\left(1-\exp \left(-a\left\langle D_{n c}-D\right\rangle\right)\right)
$$

where $\tau_{c}$ and $a$ are two material coefficients; " $\langle$.$\rangle " denotes the positive part$ operator ; $\tau_{c}$ represents the inverse of the maximum damage rate. An accurate identification of the parameters of the constitutive law, especially concerning damage, plays a key role in obtaining good results. These parameters were identified in (Suffis, 2004) and are given in Table 6.

In order to avoid mesh dependency, the mesh should verify $\Delta l<l_{c a r}$, where $l_{\text {car }}$ and $\Delta l$ denote respectively the characteristic length of the fully damaged zone and the element size. It was shown in (Suffis et al., 2003) that $l_{\text {car }}$ can be approximated by :

$$
l_{\text {car }} \simeq c \tau_{c} \ln \left(\frac{\Delta \sigma}{\sigma_{\text {lim }}}\right)
$$

where $\Delta \sigma$ is the stress jump caused by the impact and $\sigma_{\text {lim }}$ is such that :

$$
E \epsilon_{p}^{s}<\sigma_{l i m}<E\left(\epsilon_{p}^{s}+3 \frac{E \epsilon_{p}^{c}-E \epsilon_{p}^{s}}{a}\right)
$$

E being the material's Young's modulus.

$\Delta \sigma$ can be approximated through a simple analytical calculation of an idealized circular plate with no hole. As a consequence, the element size in 
the damaged zone is such that $\Delta l<1.5 \mathrm{~mm}$. In order to have several elements in the damage zone, we chose $\Delta l=0.5 \mathrm{~mm}$.

Once an element has become fully damaged, it is eroded, i.e. removed from the model. In order to prevent any loss of mass (and, therefore, of energy) during the calculations, the eroded elements are replaced by debris represented by spheres of equivalent mass. These spheres are shown in red in Figure 36.

\subsubsection{Comparison with experimental results}

Figure 36 presents the numerical model of test TF14A5 at $t=2.5 \mathrm{~ms}$. The time to fracture was determined experimentally at $t \sim 1.5 \mathrm{~ms}$ using the pressure curve, in which it corresponds to a sharp drop in the signal (see Figure 37). The pressure curve obtained with the model, shown in Figure 37, agrees well with the experimental results in terms of amplitudes, variations and time to fracture, which was also calculated at about $t \sim 1.5 \mathrm{~ms}$. Curve 38 confirms the good quality of the simulation because one can observe matching experimental and numerical deflections of the specimen at a distance $\left(D_{\text {ext }}+\right.$ d)/4 from the center of the hole.

These results confirm that the numerical model is capable of predicting post-impact phenomena accurately, including the possible rupture of the specimen.

\subsection{Test FXA5}

Finally, let us study test FXA5. The specimen used was thin $\left(e_{p}=2 \mathrm{~mm}\right)$ and included two $30 \mathrm{~mm}$ precracks forming a cross. As a result of the pressure shock, the cracks propagated; then the petals thus formed opened up 
and let the fluid escape. The state of the specimen at the end of the test is shown in Figure 39, with the cracks' paths marked in red. This test is especially interesting because it enables us to test the capability of the model to reproduce the cracking process and the resulting opening of the petals.

\subsubsection{The SPH shell model}

The FE model used previously cannot account for crack propagation and could not be used to simulate test FXA5. In this case, we studied the specimen using a shell model based on the SPH meshless method. This SPH shell model (SPHS) was developed in (Maurel and Combescure, 2008) and is especially suitable for modeling cracks, as proven in (Caleyron et al., 2011). The reader can refer to these works for a detailed description of the model. Only the main points will be reviewed here.

Formulation. The SPHS formulation is based on the Mindlin-Reissner theory and relies on the assumption that the thickness $e$ of the structure is small compared to its other dimensions. Thus, one can write :

$$
\vec{x}(t)=\vec{x}_{m}(t)+\xi \vec{n}(t) \quad \xi \in\left[-\frac{e}{2} ;+\frac{e}{2}\right]
$$

where $\vec{x}_{m}$ denotes the position of the point in the mean plane of the shell and $\vec{n}$ is the pseudo-normal which materializes the orientation of the material. The theory takes into account transverse shear, so $\vec{n}$ is not necessarily normal to the mean plane.

The original SPH method (Gingold and Monaghan, 1977) suffers from a numerical instability known as tension instability. Indeed, the authors of (Wen et al., 1994) showed that the Eulerian kernel used classically is unstable in the presence of tension stresses. The solution chosen for the SPHS 
model was taken from (Belytschko et al., 2000) and consists in using a total Lagrangian formulation whose kernel is stable.

Therefore, the SPH shell is studied using the total Lagrangian formalism, in which the membrane, transverse shear and bending strains are calculated. Then, the application of a plane stress constitutive relation leads to the corresponding stresses. These stresses are integrated across the thickness in order to obtain the stress resultants of the shell. Finally, the equilibrium of the shell is expressed as a function of the generalized membrane loads $\underline{\underline{\mathrm{N}}}$ and transverse shear loads $\vec{T}$ and of the bending moments $\underline{\underline{M}}$.

Discretization. The global response of the structure is described in its mean plane, which is discretized using a single layer of SPH nodes. Each node can be viewed as a cylinder with its axis normal to the shell, its thickness $e$ and its radius chosen as a function of the desired mesh fineness. The nodes have 5 degrees of freedom because the "drilling rotation" is not taken into account (see Figure 40).

The spatial discretization requires the introduction of shape functions allowing the representation and the derivation of a data field. In order to do that, we use MLS functions as described in (Dilts, 1999, 2000). These functions are built from an $n^{\text {th }}$-order polynomial basis, which gives them $n^{\text {th }}$ order consistency. Second-order functions are used for the approximation of the field of the pseudo-normals $\vec{n}$ in order to achieve a good representation of the curvature of the shell. First-order functions are used for the derivation operations. The reader can refer to (Dilts, 1999; Belytschko et al., 1994b) for details concerning the construction of these functions.

Finally, the use of collocation for strong formulations such as the SPH 
method leads to the occurrence of zero-energy modes. (Belytschko et al., 2000) presented a complete study of this problem and showed that the use of a total Lagrangian formulation reduces these instabilities. However, this is not sufficient in the case of the SPH model because the field of the normals to the mean surface is sensitive to the presence of instabilities affecting the curvature of the shell. Since the problem comes from the fact that the kinematic fields and the strain and stress fields are supported by the same nodes, (Belytschko et al., 2000; Dyka et al., 1997) proposed the introduction of a second type of nodes called Stress Points (SP). No material is attached to these points, which play a role similar to that of Gauss points in the FEM : the strain and stress fields are calculated at the SPs using the kinematic quantities at the nodes, then the equilibrium equations are written back at the nodes again. The number and the positions of the SPs are parameters of the method.

Consequently, the constitutive relation of the material is applied at the model's SPs. The law used for test FXA5 was identical to that presented in Section 5.3.1. The difference resided in the use of a global model based on Ilyushin's theory (Ilyushin, 1956) because only one SP was used through the thickness of the shell.

Fracture. The SPHS model was extended to the modeling of fracture in (Caleyron et al., 2011). When the damage calculated at a particular SP reaches its critical value $D_{c}$, the Elementary Representative Volume (ERV) supported by the SP is considered to be fractured. Fully damaged ERVs are treated as strong discontinuities, which is an efficient way to address cases where the crack's lips separate significantly. The method we implemented consists of four stages : 
- application of the free edge condition to the neighboring nodes of the cracked ERV ;

- elimination of the links cutting the cracked ERV by means of the visibility method (Belytschko et al., 1994a),

- treatment of the fragments, i.e. the nodes which no longer have neighbors ;

- updating of the MLS functions.

Thus, in this model, a crack is represented by a series of cracked ERVs, as shown in Figure 41. These ERVs are supported by the SPs of the model. This method is attractive because it does not require an explicit representation of the cracks, which enables one to deal with multicracking and crack branching easily.

\subsubsection{Comparison with experimental data}

The SPHS model presented previously was introduced locally over the specimen's mesh through a coupling with the Q4GS elements, as shown in Figure 42. This coupling was of the Arlequin type : the equality of velocities was enforced weakly over a common zone called the overlapping zone, in which the energies of the two models are blended. The theory is not described in this paper. The reader can refer to (Ben Dhia and Rateau, 2004; Bauman et al., 2008; Chuzel-Marmot et al., 2011; Caleyron, 2011) for further details. The FE and SPHS models used the same constitutive law, which was that used for test TF14A5 (see Section 5.3), i.e. a Von Mises' plasticity law coupled with Lemaitre and Chaboche's damage : the model was global for the SPHS and integrated through the thickness with 5 Gauss points for the finite elements. The contact between the SPH shell and the SPH fluid model 
was handled through the pinball method. The other contacts were handled through the sliding surface method. The SPHS discretization required about 15,000 nodes and SPs with a radius $R_{s p h}=0.35 \mathrm{~mm}$.

Figure 43 shows the state of the model at $t=2.1 \mathrm{~ms}$. The behavior was satisfactory because one can indeed observe a propagation of the cracks and the associated opening of the petals.

The experimental and numerical crack paths and sizes are compared in Figure 44. The crack lengths appear to be slightly overestimated, due probably to the coarse fluid discretization exaggerating the opening of the shell. Nevertheless, the simulated paths are consistent with those observed experimentally since, in both cases, the cracks propagated in mixed mode. These curved paths led to the opening of two of the four petals, marked with a "+" sign on the figure. One should also note the branching of the two cracks, which was not observed experimentally. This branching seems to occur near the overlapping zone of the Arlequin coupling, which suggests that the SPHS domain used was a little too small, leading to nonlinear phenomena in the overlapping zone which may have been responsible for errors in the crack's propagation.

Even though a more refined fluid discretization could have led to better results, the comparison of the numerical and experimental results is satisfactory. The model we implemented enables one to predict effectively the fracture of a tank subjected to impact and the associated leakage flow. 


\section{Conclusions}

This paper presented and studied an experiment which is representative of an impact on a fluid-filled tank. The results of this experiment were compared with those of a numerical model.

First, the study of tests which presented no fracture or crack propagation gave us an understanding of the phenomena involved in the experiment. In particular, we showed that the impacted tank behaves as a true oscillator in which the fluid and structural phenomena are coupled in a nonpermanent way. Consequently, the prediction of the behavior of an impacted tank requires a precise modeling of the fluid, the thin structure and their interactions. The numerical model we proposed studies the fluid using the SPH meshless method. This method is especially suitable for impacts involving fluids with significant free surface variations. In addition, the main structural elements (the cylinder, the piston and the specimen) were modeled in order to represent the stiffness of the experimental setup accurately. Finally, the nonpermanent fluid-structure interactions were handled by means of the sliding surface method. The numerical results obtained were satisfactory, particularly concerning the prediction of the quantities of interest immediately following the impact. The fluid outflow velocity, the displacement of the carriage and the deflection of the specimen were correctly predicted by the model. The pressure peak which immediately followed the impact was correctly estimated, too, which is an important point with regard to the prediction of the failure of the tank. However, once the flow had become steady, the pressure in the cylinder was incorrectly predicted since the calculated values were up to twice those measured experimentally. These inaccuracies 
are due to the fluid model, which is suitable for impact analysis, but less effective in the case of stationary flows. Then, more complex tests involving the propagation of cracks and, in some cases, the complete rupture of the specimen were studied. We started out by showing that the model is capable of predicting the complete failure of the tank accurately. In order to do that, we used a damageable elastic-plastic constitutive relation with a delay effect. An accurate identification of the parameters, especially the delay effect, was found to be a key factor in obtaining good results. Finally, we studied a test presenting a more complex crack path. In this case, the FE model was replaced by a meshless SPH shell model dedicated to the modeling of cracks. This model was introduced locally in the zone where fracture was expected, which enabled us to achieve reasonable computation times.

In conclusion, an impacted tank behaves like a true fluid-structure oscillator. Therefore, it is necessary to model both the structural and fluid phenomena accurately, including the most complex of these phenomena which are damage, fracture and significant free surface variations. In this context, the use of meshless methods appears to be particularly attractive. Finally, the fluid-structure coupling method must be capable of handling nonpermanent interactions.

\section{Acknowledgements}

The authors wish to thank the French Atomic Energy Commission (CEA) and the French Electricity Company (EDF) for their support regarding the EUROPLEXUS software; ONERA for its experimental support; and the EEC for its financial support concerning the test rig. F. Caleyron extends 
his personal thanks to Jacky Fabis of ONERA Lille for helpful discussions.

\section{Références}

Allix, O., Deu, J., 1997. Delayed-damage modelling for fracture prediction of laminated composites under dynamic loading. Engineering Transactions/Rozprawy Inzynierskie 45, 29-46.

Anderson, C., et al., 1999. Simulation and analysis of a 23-mm hei projectile hydrodynamic ram experiment. International journal of impact engineering 22, 981-997.

Bauman, P., Ben Dhia, H., Elkhodja, N., Oden, J., S., P., 2008. On the application of the arlequin method to the coupling of particle and continuum models. Computational Mechanics 42, 511-530.

Bazoant, Z., Belytschko, T., 1985. Wave propagation in a strain-softening bar : exact solution. Journal of engineering mechanics 111, 381-389.

Belytschko, T., Gu, L., Lu, Y., 1994a. Fracture and crack growth by element free galerkin methods. Modelling and Simulation in Materials Science and Engineering 2, 519.

Belytschko, T., Guo, Y., Liu, W., Xiao, S., 2000. A unified stability analysis of meshless particle methods. International Journal for Numerical Methods in Engineering 48, 1359-1400.

Belytschko, T., Lu, Y., Gu, L., 1994b. Element-free galerkin methods. International journal for numerical methods in engineering 37, 229-256. 
Belytschko, T., Neal, M., 1991. Contact-impact by the pinball algorithm with penalty and lagrangian methods. International Journal for Numerical Methods in Engineering 31, 547-572.

Ben Dhia, H., Rateau, G., 2004. The arlequin method as a flexible engineering design tool. International Journal For Numerical Methods in Engineering $62,1442-1462$.

Caleyron, F., 2011. Simulation numérique par la méthode SPH de fuites de fluide consécutives à la déchirure d'un réservoir sous impact. Ph.D. thesis. Université de Lyon.

Caleyron, F., Combescure, A., Faucher, V., Potapov, S., 2011. Dynamic damage-fracture transition simulation for sph shells. International Journal for Numerical Methods in Engineering in press.

Casadei, F., 1973. A General Impact-Contact Algorithm based on hierarchic pinballs for the EUROPLEXUS software system. Technical Report.

Chao, T., Shepherd, J., 2005. Fracture response of externally flawed aluminum cylindrical shells under internal gaseous detonation loading. International journal of fracture 134, 59-90.

Chuzel-Marmot, Y., Ortiz, R., Combescure, A., 2011. Three dimensional sphfem gluing for simulation of fast impacts on concrete slabs. Computers \& Structures .

Colagrossi, A., Landrini, M., 2003. Numerical simulation of interfacial flows by smoothed particle hydrodynamics. Journal of Computational Physics 191, 448-475. 
Dalrymple, R., Rogers, B., 2006. Numerical modelling of water waves with the sph method. Coastal Engineering 53, 141-147.

Dilts, G., 1999. Moving-least-squares-particle hydrodynamics i. consistency and stability. International Journal for Numerical Methods in Engineering $44,1115-1155$.

Dilts, G., 2000. Moving least-squares particle hydrodynamics ii : conservation and boundaries. International Journal for Numerical Methods in Engineering 48, 1503-1524.

Disimile, P., Swanson, L., Toy, N., 2009. The hydrodynamic ram pressure generated by spherical projectiles. International Journal of Impact Engineering 36, 821-829.

Dyka, C., Randles, P., Ingel, R., 1997. Stress points for tension instability in sph. International journal for numerical methods in engineering 40, $2325-2341$.

Freitas, C., Anderson Jr, C., Goodlin, D., 1997. Hydrodynamic Ram : A Review of Experimental Data for Use in Validation of Numerical Simulations. Technical Report. SwRI Report 06-7365/001, Southwest Research Institute, San Antonio, TX 78228, prepared for Wright Laboratory, WrightPatterson AFB, OH.

Freitas, C., Anderson Jr, C., Walker, J., Littlefield, D., 1996. Hydrodynamic ram : a benchmark suite, structures under extreme loading conditions, pvp, vol. 325. New York : ASME, 63-74. 
Gingold, R., Monaghan, J., 1977. Smoothed particle hydrodynamics-theory and application to non-spherical stars. Monthly Notices of the Royal Astronomical Society 181, 375-389.

Gray, J., Monaghan, J., Swift, R., 2001. Sph elastic dynamics. Computer methods in applied mechanics and engineering 190, 6641-6662.

Hallquist, J., Goudreau, G., Benson, D., 1985. Sliding interfaces with contactimpact in large-scale lagrangian computations. Computer Methods in Applied Mechanics and Engineering 51, 107-137.

Ilyushin, A., 1956. Plasticité. Eyrolles.

Keesecker, A., Dávila, C., Johnson, E., Starnes, J., et al., 2003. Crack path bifurcation at a tear strap in a pressurized shell. Computers \& structures $81,1633-1642$.

Ladeveze, P., 1991. About a damage mechanics approach. Mechanics and Mechanisms of Damage in Composites and Multi-materials 35, 119-141.

Ladevèze, P., 1992. A damage computational method for composite structures. Computers \& structures 44, 79-87.

Lee, E.S., Moulinec, C., Xu, R., Violeau, D., Laurence, D., Stansby, P., 2006. Comparisons of weakly compressible and truly incompressible sph algorithms for 2d flows. Journal of Computational Physics 227, 8417-8436.

Lee, E.S., Violeau, D., Issa, R., Ploix, S., 2010. Application of weakly compressible and truly incompressible sph to 3 -d water collapse in waterworks. Journal of Hydraulic Research 48, 50-60. 
Lemaitre, J., Chaboche, J., Germain, P., 1985. Mécanique des matériaux solides. Dunod.

Maurel, B., Combescure, A., 2008. An sph shell formulation for plasticity and fracture analysis in explicit dynamics. International Journal for Numerical Methods in Engineering 76, 949-971.

Maurel, B., Potapov, S., Fabis, J., Combescure, A., 2009. Full sph fluidshell interaction for leakage simulation in explicit dynamics. International Journal for Numerical Methods in Engineering 80, 210-234.

Monaghan, J., Gingold, R., 1983. Shock simulation by the particle method sph. Journal of Computational Physics 52, 374-389.

Needleman, A., 1988. Material rate dependence and mesh sensitivity in localization problems. Computer Methods in Applied Mechanics and Engineering $67,69-85$.

Potapov, S., Maurel, B., Combescure, A., Fabis, J., 2009. Modeling accidental-type fluid-structure interaction problems with the sph method. Computers \& Structures 87, 721-734.

Rabczuk, T., Areias, P., Belytschko, T., 2007. A meshfree thin shell method for non-linear dynamic fracture. International Journal for Numerical Methods in Engineering 72, 524-548.

Rabczuk, T., Gracie, R., Song, J., Belytschko, T., 2009. Immersed particle method for fluid-structure interaction. International Journal for Numerical Methods in Engineering 81, 48-71. 
Sauer, M., 2010. Simulation of high velocity impact in fluid-filled containers using finite elements with adaptive coupling to smoothed particle hydrodynamics. International Journal of Impact Engineering .

Suffis, A., 2004. Développement d'un modèle d'endommagement à taux de croissance contrôlé pour la simulation robuste de ruptures sous impacts. Ph.D. thesis. Université de Lyon.

Suffis, A., Lubrecht, T., Combescure, A., 2003. Damage model with delay effect : : Analytical and numerical studies of the evolution of the characteristic damage length. International journal of solids and structures 40, $3463-3476$.

Timm, T., 2003. Beschuss von flüssigkeitsgefüllten Behältern. Ph.D. thesis. Karlsruhe University, Germany.

Varas, D., López-Puente, J., Zaera, R., 2009a. Experimental analysis of fluidfilled aluminium tubes subjected to high-velocity impact. International Journal of Impact Engineering 36, 81-91.

Varas, D., Zaera, R., López-Puente, J., 2009b. Numerical modelling of the hydrodynamic ram phenomenon. International Journal of Impact Engineering $36,363-374$.

Wen, Y., Hicks, D., Swegle, J., 1994. Stabilizing SPH with conservative smoothing. Technical Report. Sandia National Labs., Albuquerque, NM (United States). 


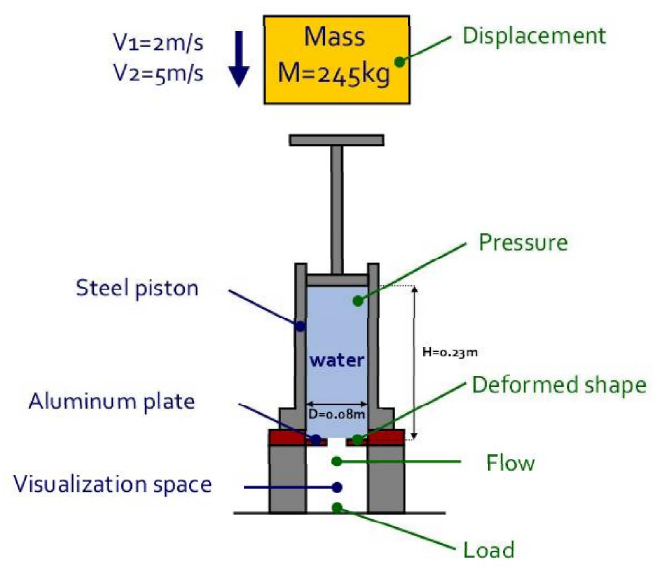

(a) Diagram

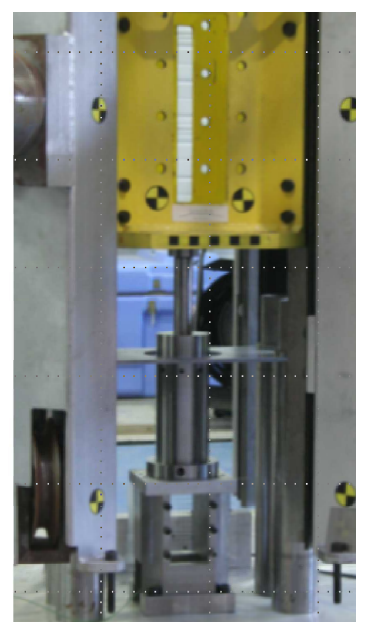

(b) Photograph

Figure 1: The experimental setup

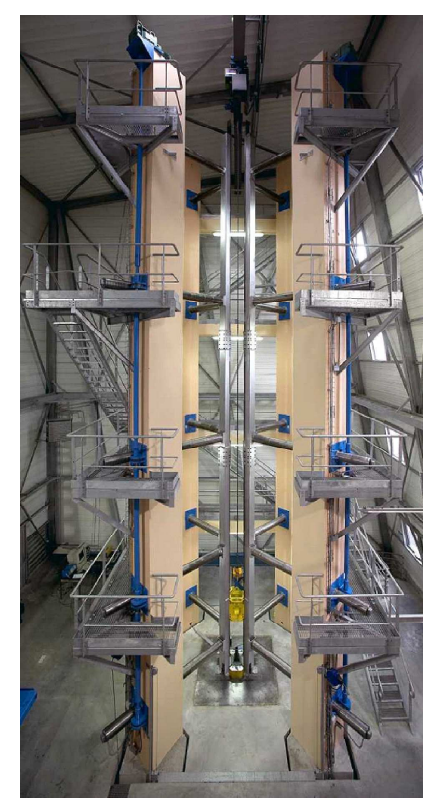

Figure 2: The drop tower of ONERA Lille 


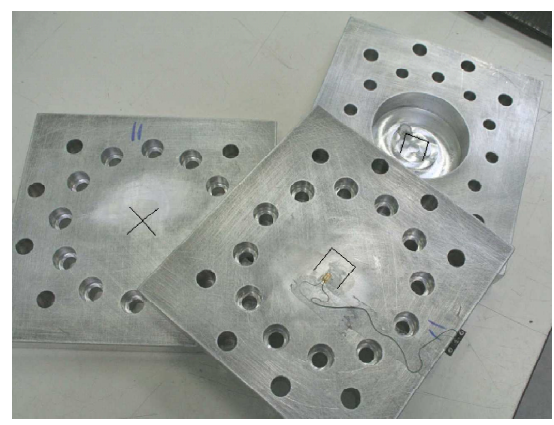

FigurE 3: Examples of specimens before impact : X-shaped and U-shaped precracks

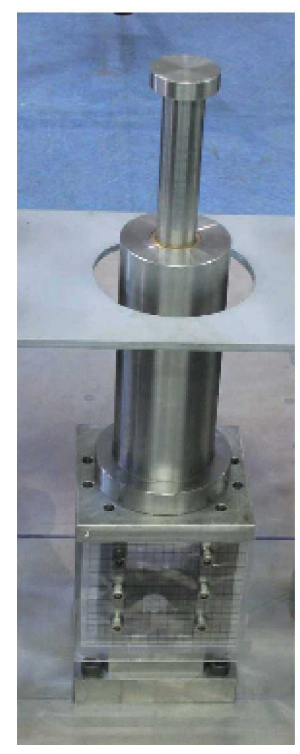

(a) Photograph

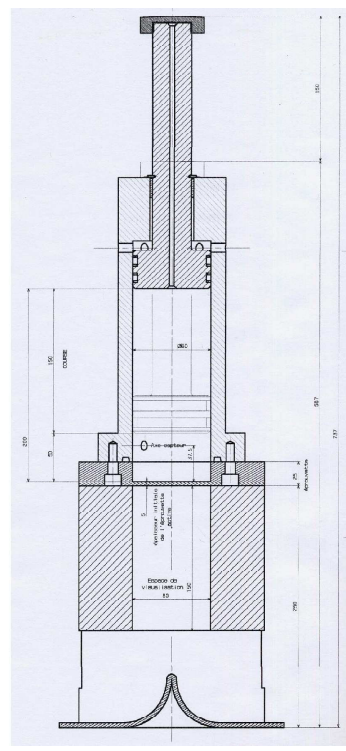

(b) General diagram

FiguRE 4: Overview of the experimental device 


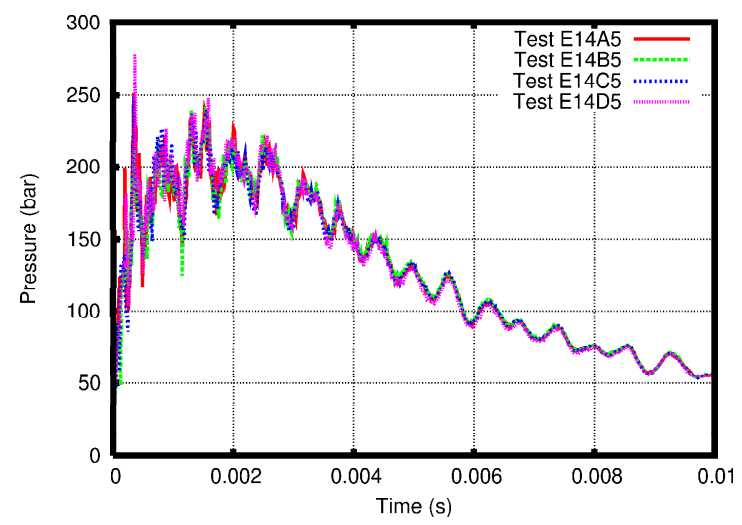

Figure 5: The pressure in the cylinder (tests E14A-B-C-D5)

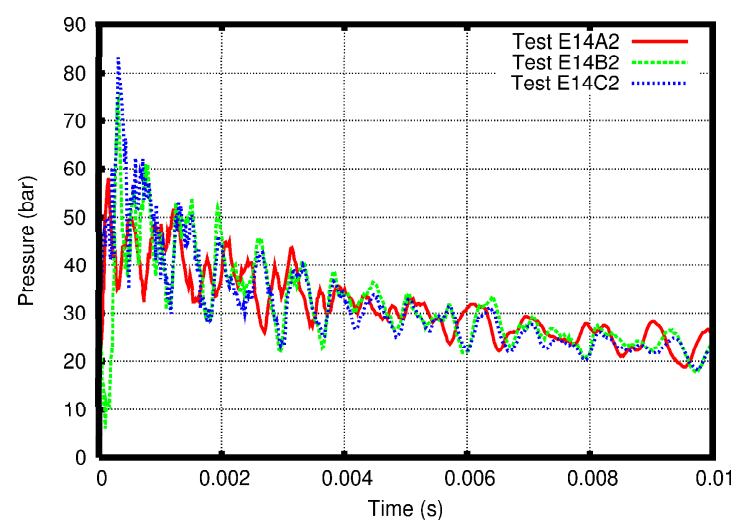

Figure 6: The pressure in the cylinder (tests E14A-B-C2)

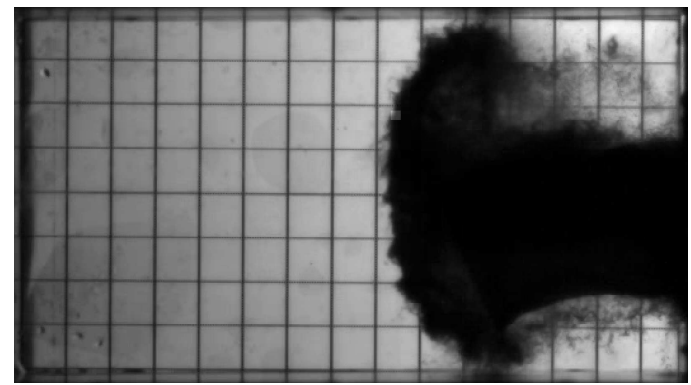

Figure 7: The outflow at $t=2.5 \mathrm{~ms}$ (test E20A2) 


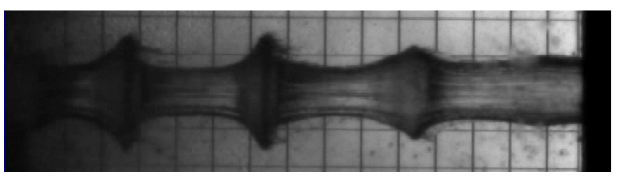

(a) $t=14.25 \mathrm{~ms}$

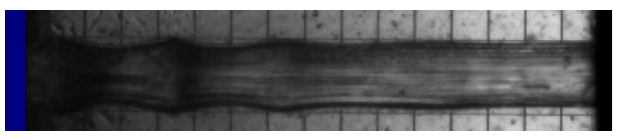

(c) $t=40.5 \mathrm{~ms}$

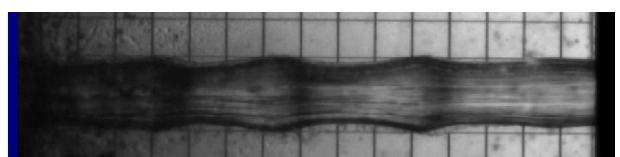

(b) $t=22.75 \mathrm{~ms}$

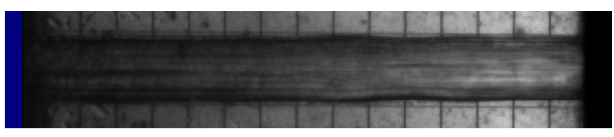

(d) $t=52.75 \mathrm{~ms}$

Figure 8: Profile of the outflow (test E20A2)

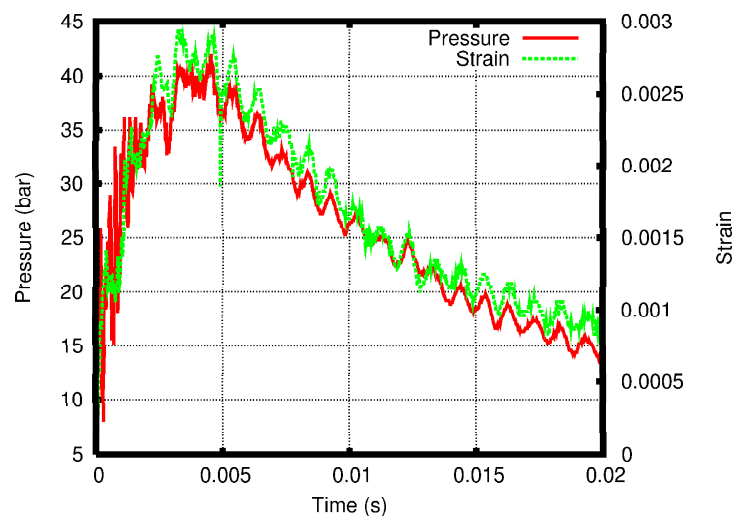

FiguRE 9: The pressure in the cylinder and deflection of the specimen (test F14A2) 


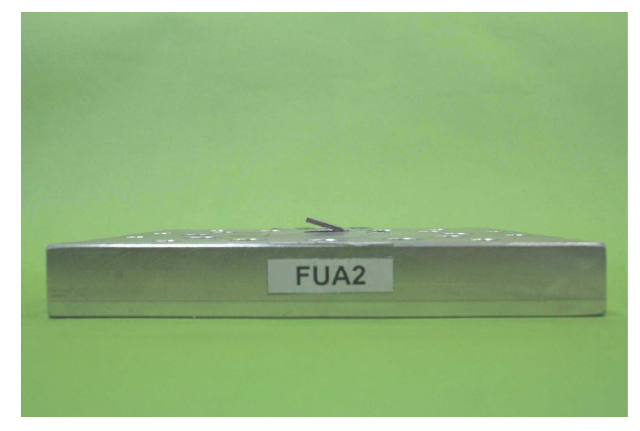

(a) test FUA2

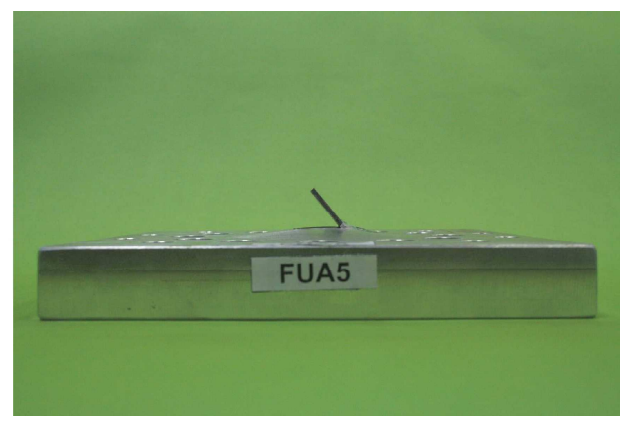

(b) test FUA5

FigURE 10: Opening of the precrack (tests FUA2 and FUA5)

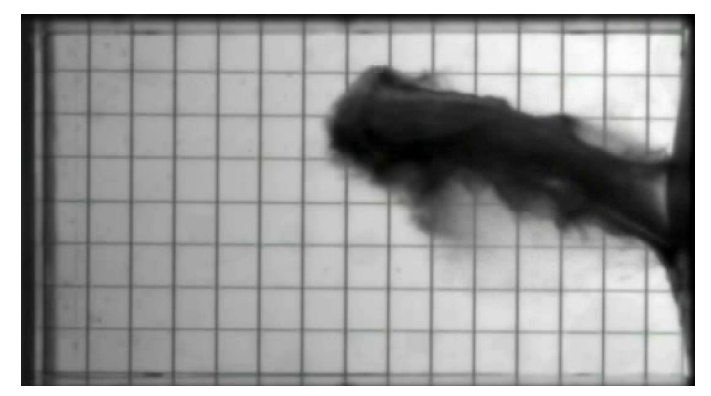

Figure 11: The outflow at $t=1.75 \mathrm{~ms}$ (test FUA5)

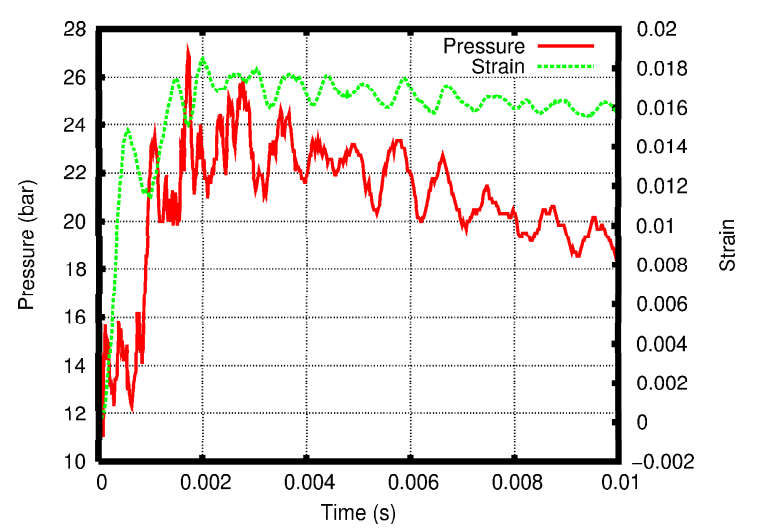

Figure 12: The pressure in the cylinder and deflection of the specimen (test FUA2) 


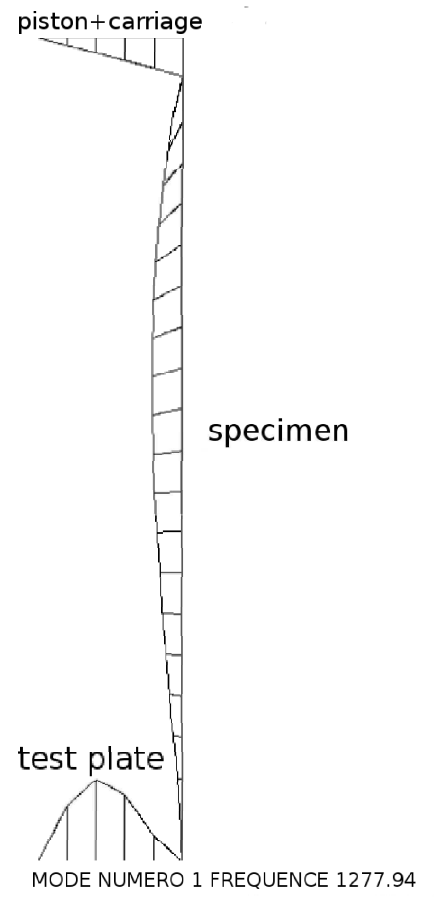

(a) Deflection mode

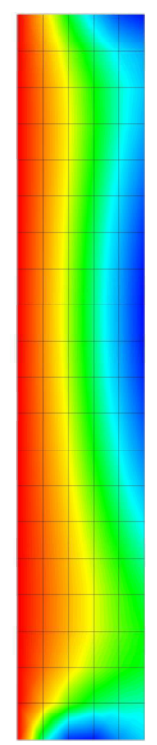

(b) Isopressure map

Figure 13: Calculation of the first mode of the water-filled piston (CASTEM) 




(a) test TF14A5

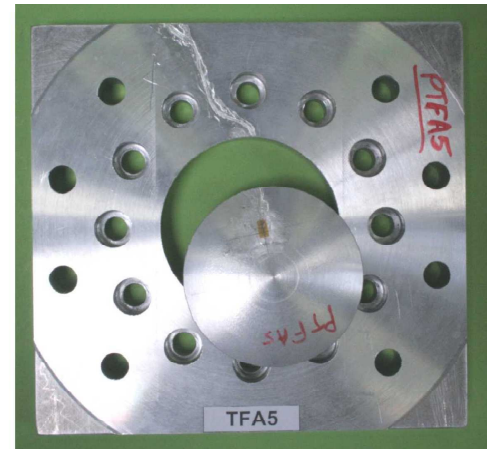

(b) test TFA5

Figure 14: Complete rupture of the bottom (tests TF14A5 and TFA5)

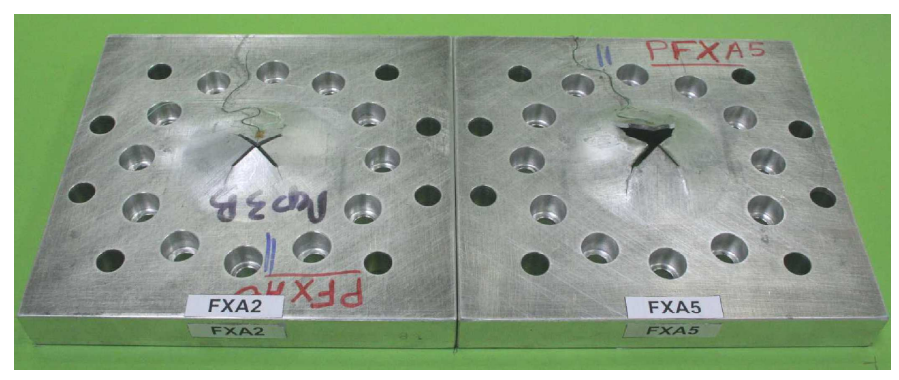

(a) tests FXA2 and FXA5

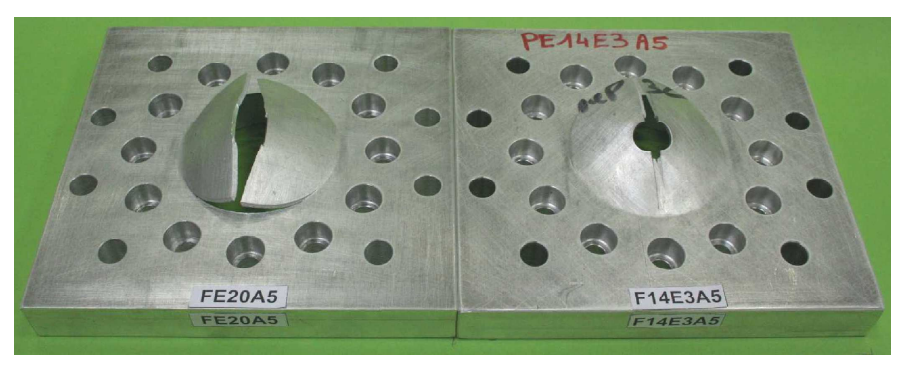

(b) tests FE20A5 and F14E3A5

FiguRE 15: Propagation of the precracks 


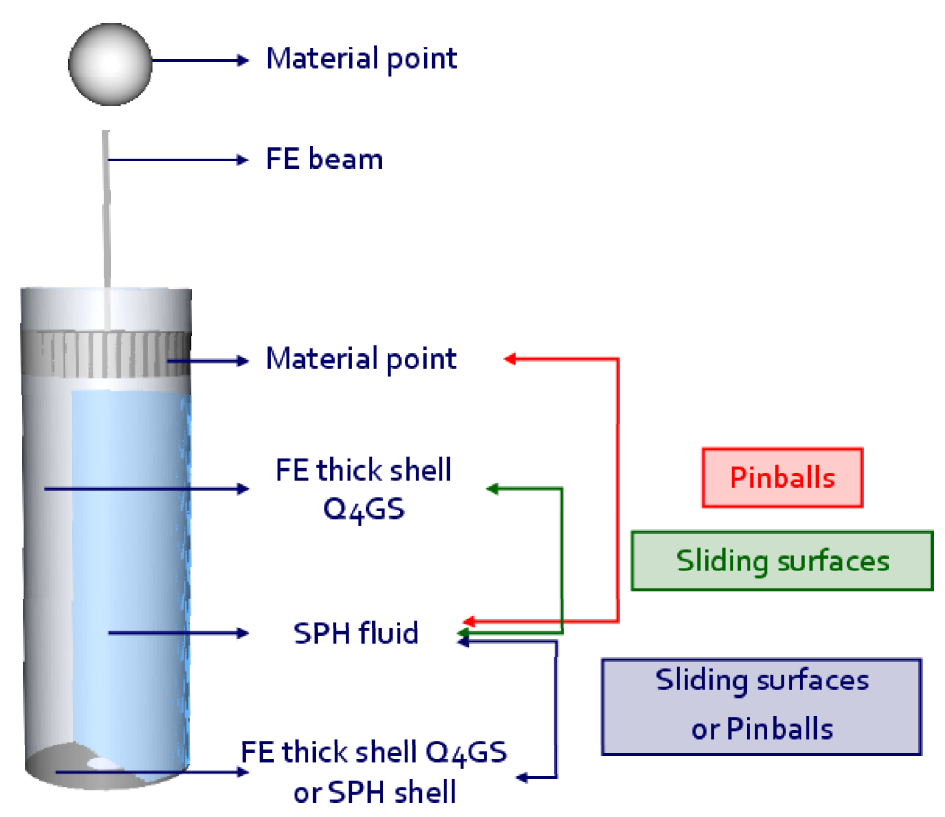

Figure 16: The numerical model of the experimental device

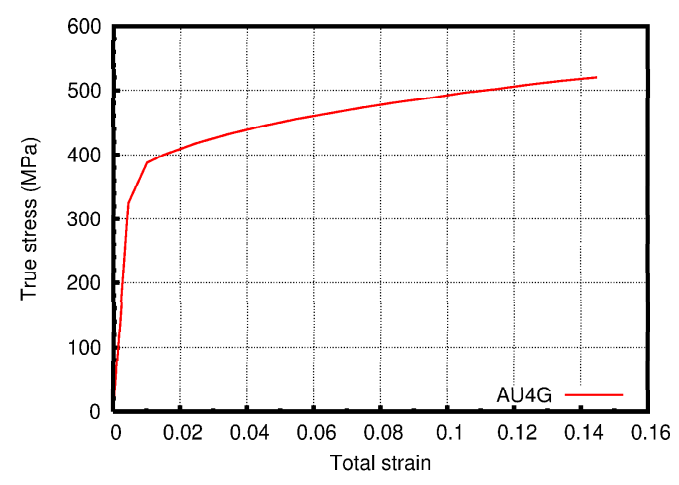

\begin{tabular}{lc}
\hline Parameter & Value \\
\hline $\mathrm{E}(\mathrm{GPa})$ & 73.1 \\
$\rho\left(\mathrm{kg} / \mathrm{m}^{3}\right)$ & 2,780 \\
$\nu$ & 0.33 \\
$\sigma_{y}(M P a)$ & 325 \\
\hline
\end{tabular}

FIGURE 17: AU4G traction curve AU4G 


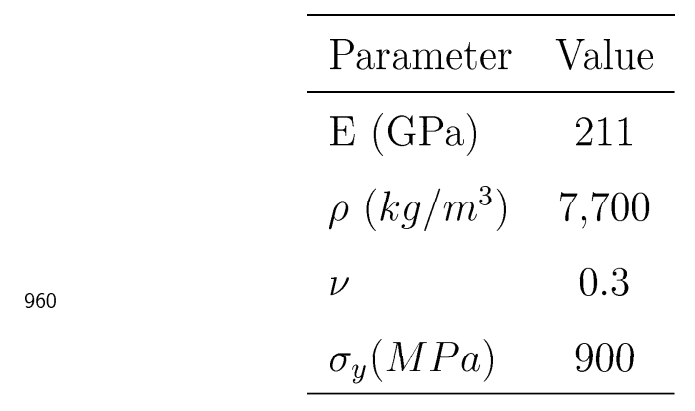

\begin{tabular}{ll}
\hline Parameter & Value \\
\hline$\rho\left(\mathrm{kg} / \mathrm{m}^{3}\right)$ & 1000 \\
$\mathrm{c}(\mathrm{m} / \mathrm{s})$ & 1450 \\
\hline
\end{tabular}

TABLE 3: The material parameters of

TABLE 2: The material parameters ofwater

APX



FiguRE 18: Modeling of the specimen's support

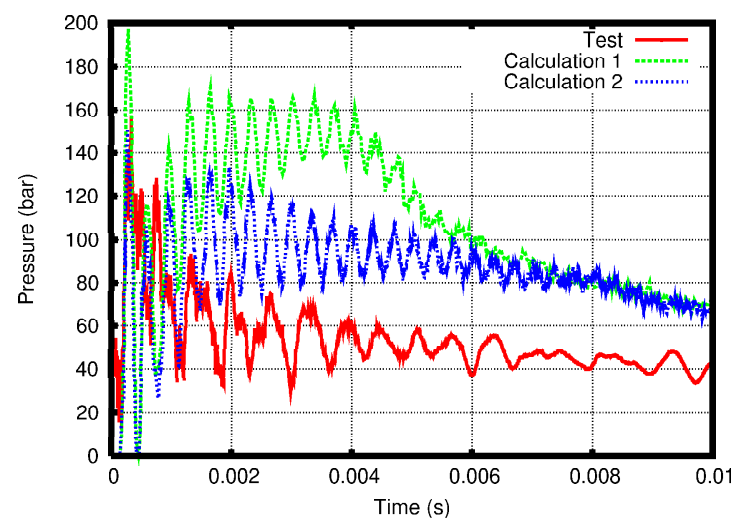

Figure 19: The pressure in the cylinder (test E20A5) 


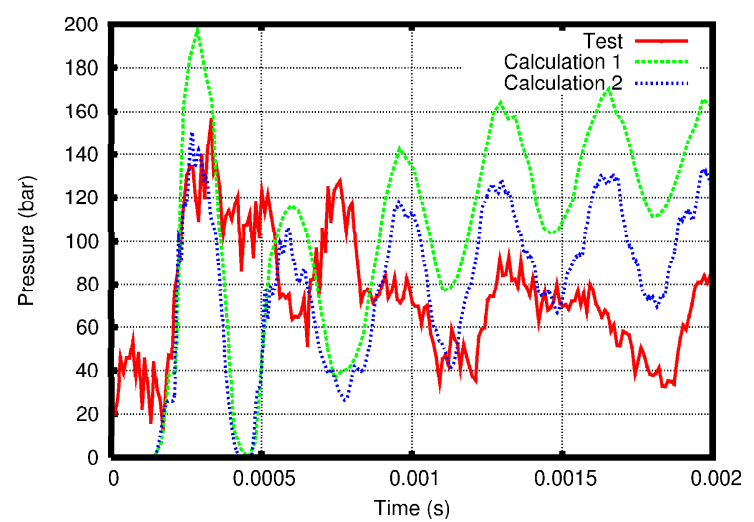

Figure 20: The pressure in the cylinder : zoom (test E20A5)

Remark : the pressure sensor is modeled using a simple FE node. The pressure is obtained by dividing the load applied by the fluid at the node by the area of an element.

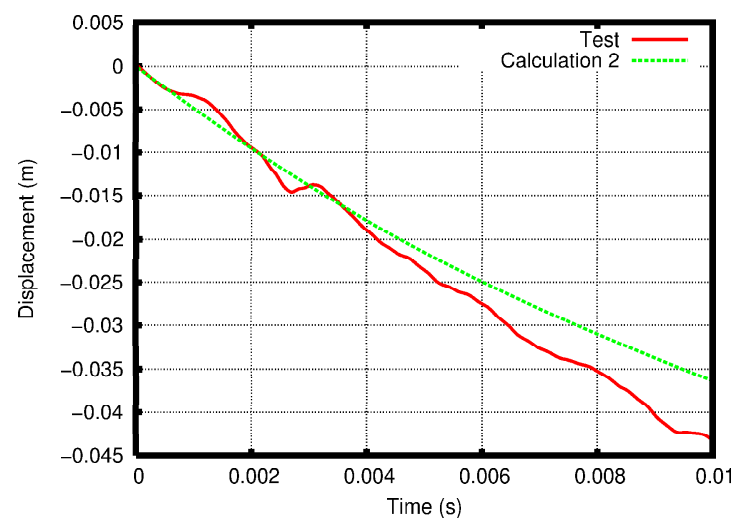

Figure 21: The displacement of the carriage (test E20A5) 


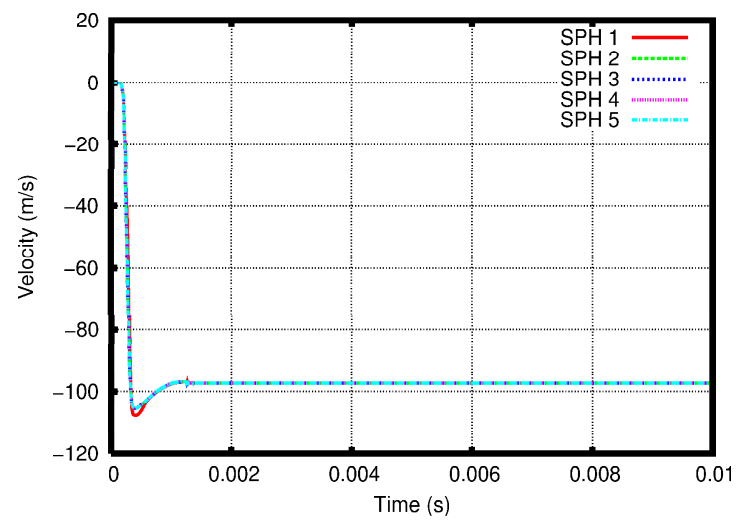

Figure 22: Outgoing velocity of the jet (test E20A5, Calculation 2)
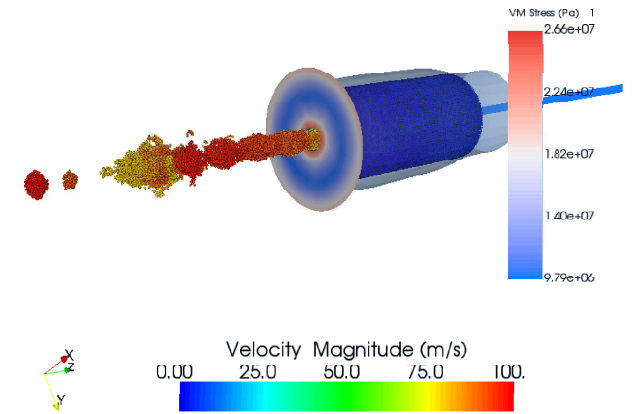

(a) Velocity, Von Mises' stress and deflection of the cylinder $(\times 1,000)$

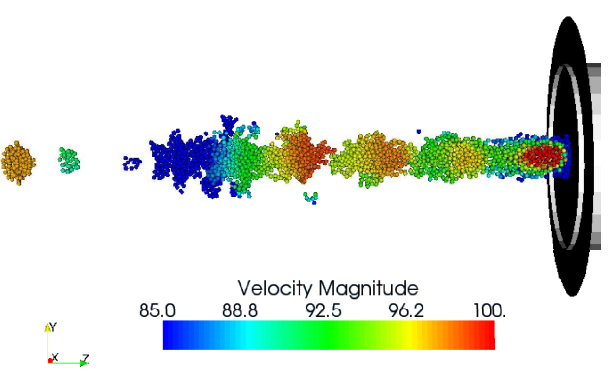

(b) Bulbs in the fluid flow

Figure 23: The simulation of test E20A5 at $t=3 \mathrm{~ms}$ (Calculation 2) 


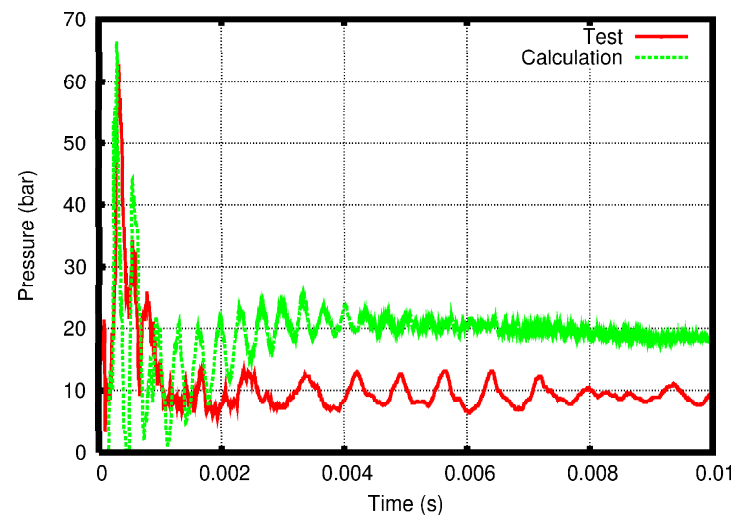

Figure 24: The pressure in the cylinder (test E20A2)

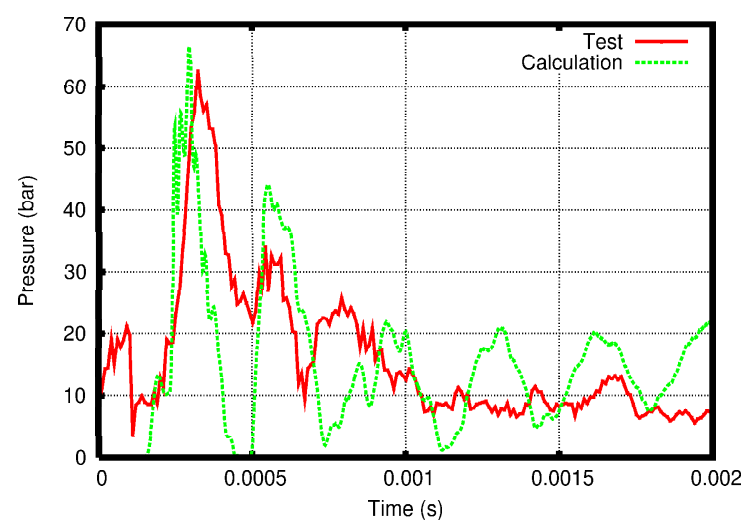

Figure 25: The pressure in the cylinder : zoom (test E20A2) 


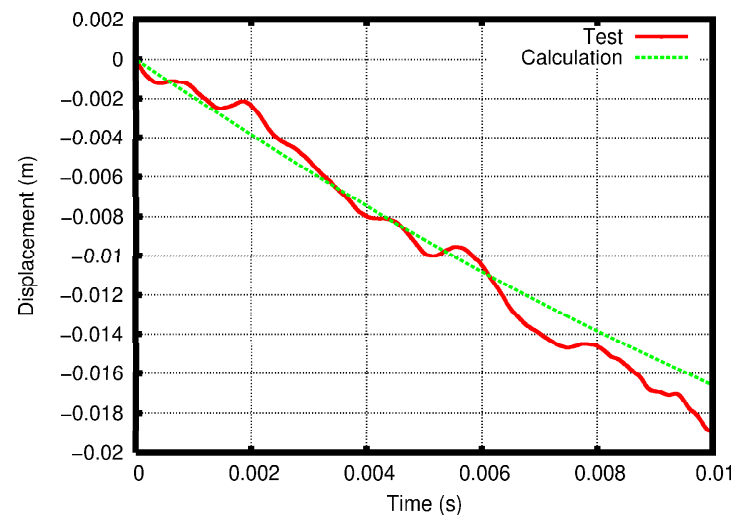

FiguRE 26: The displacement of the carriage (test E20A2)

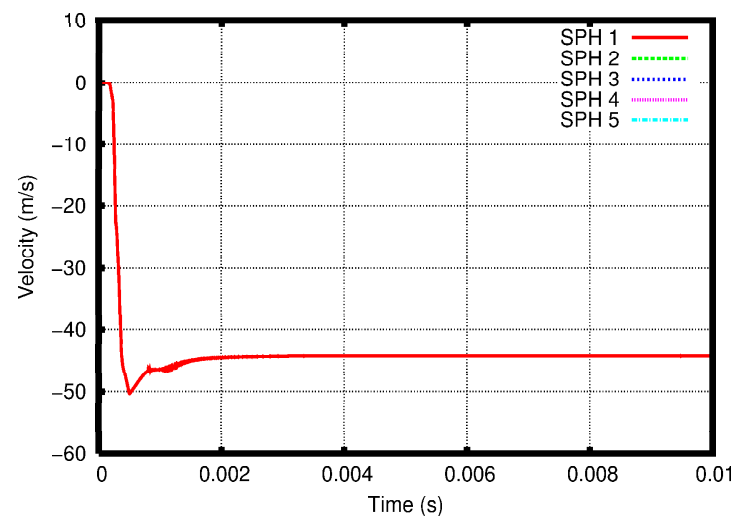

FiguRE 27: Outgoing velocity of the jet (test E20A2) 


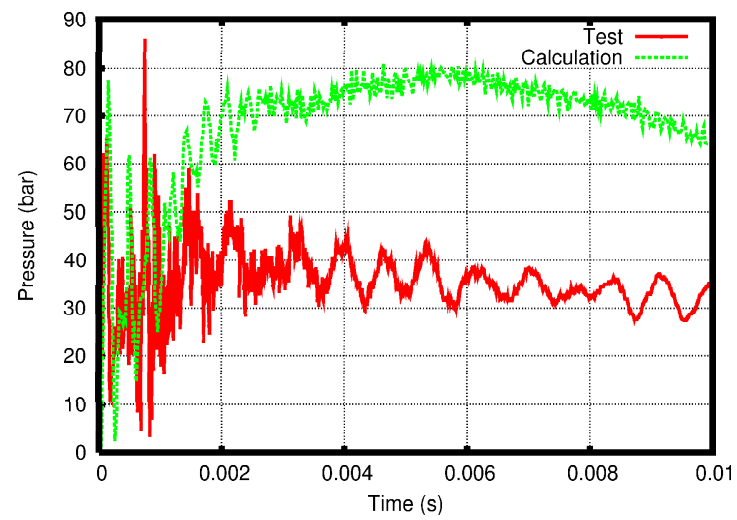

Figure 28: The pressure in the cylinder (test FUA5)

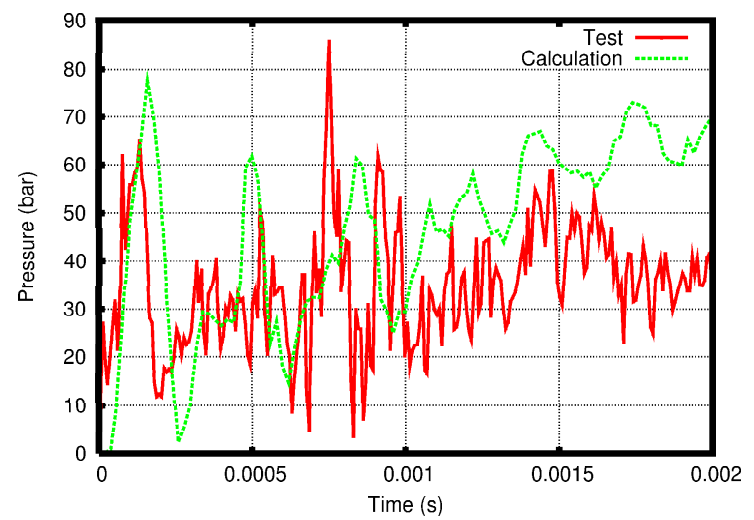

FIgURE 29: The pressure in the cylinder : zoom (test FUA5) 

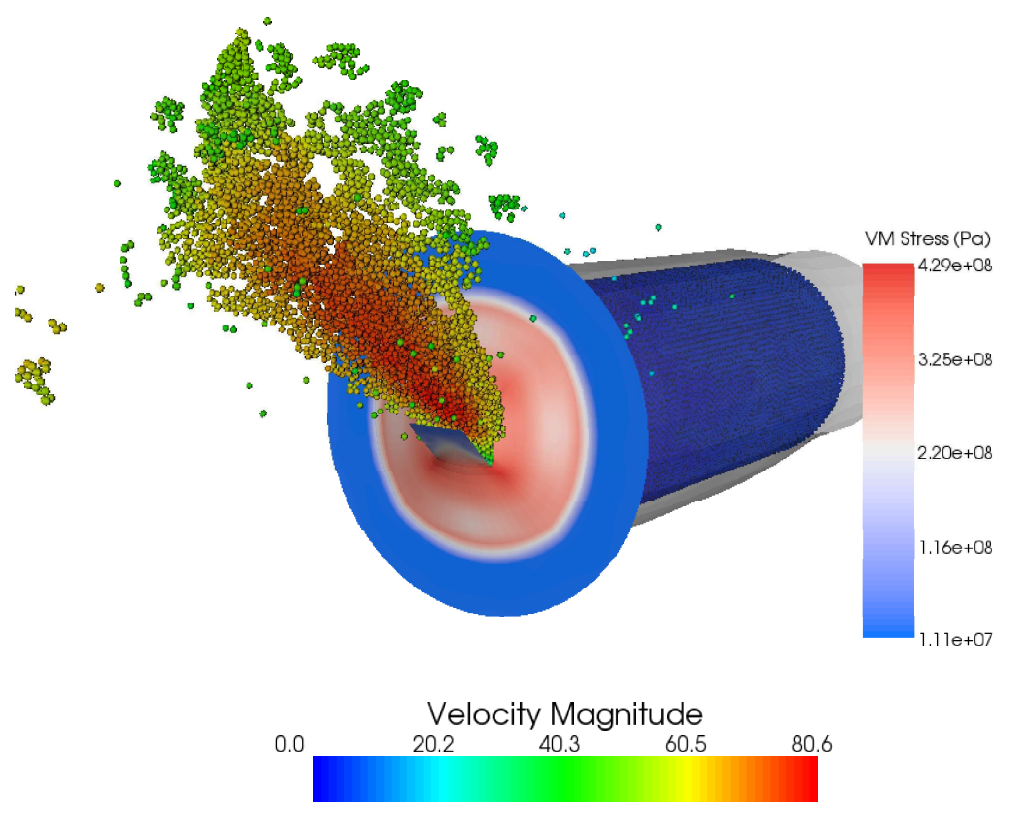

FIgURE 30: The simulation of test FUA5 at $t=3.5 \mathrm{~ms}$ 


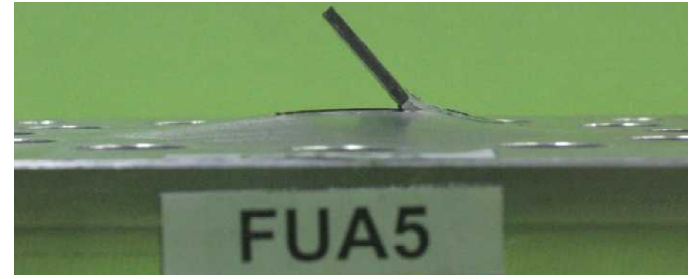

(a) Experimental deflection, $\alpha \sim 50^{\circ}$

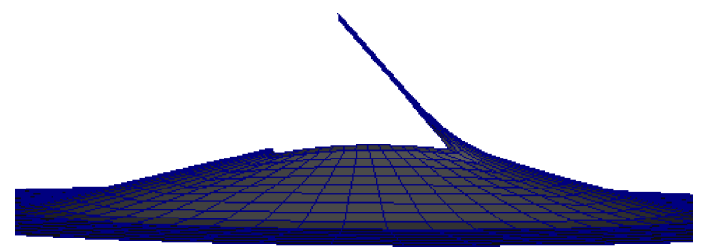

(b) Numerical deflection at $t=10 \mathrm{~ms}, \alpha \sim 50^{\circ}$

FiguRE 31: Comparison of the deflections (test FUA5)

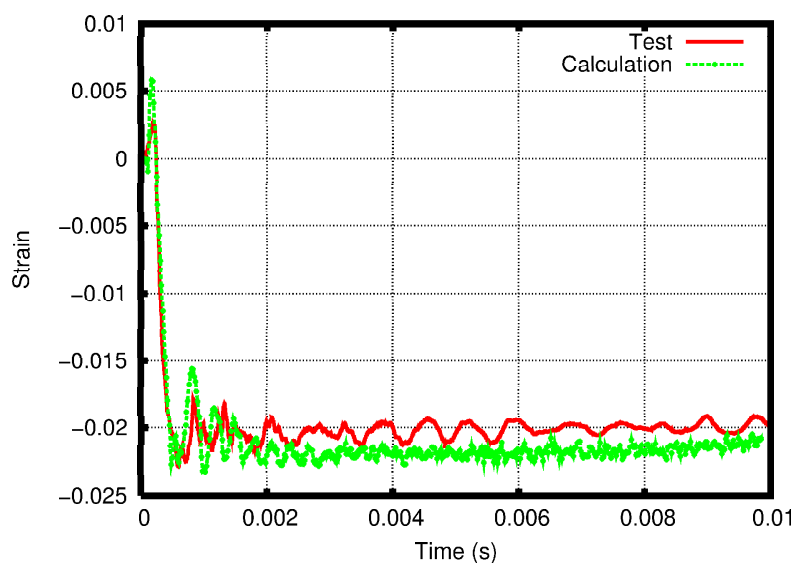

FiguRE 32: Comparison of the strains (test FUA5) 


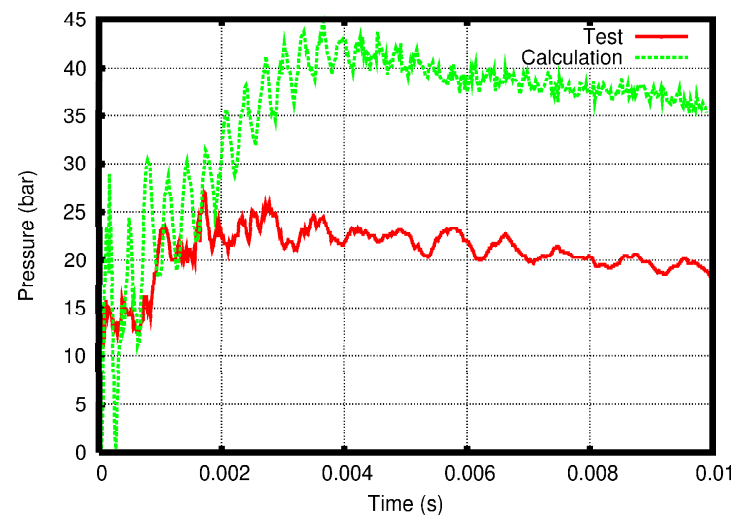

Figure 33: The pressure in the cylinder (test FUA2)

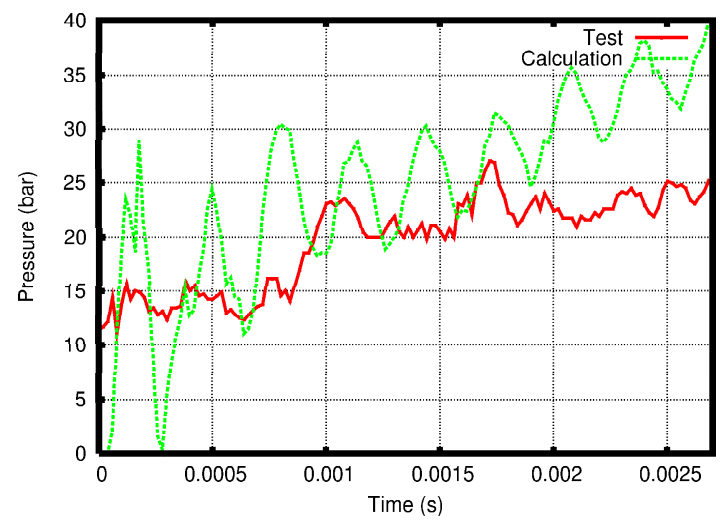

Figure 34: The pressure in the cylinder : zoom (test FUA2) 


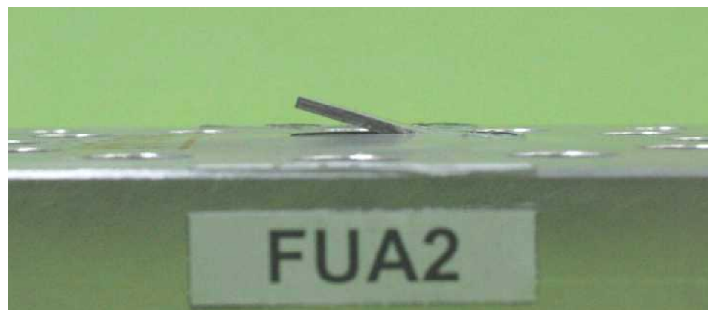

(a) Experimental deflection, $\alpha \sim 20$

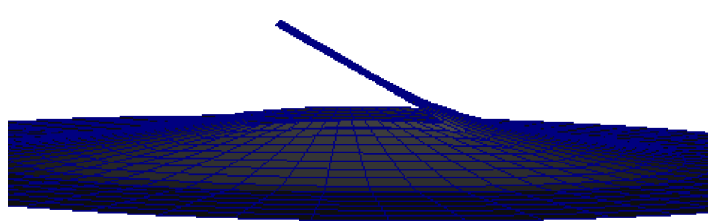

(b) Numerical deflection at $t=10 \mathrm{~ms}, \alpha \sim 28^{\circ}$

Figure 35: Comparison of the deflections (test FUA2) 


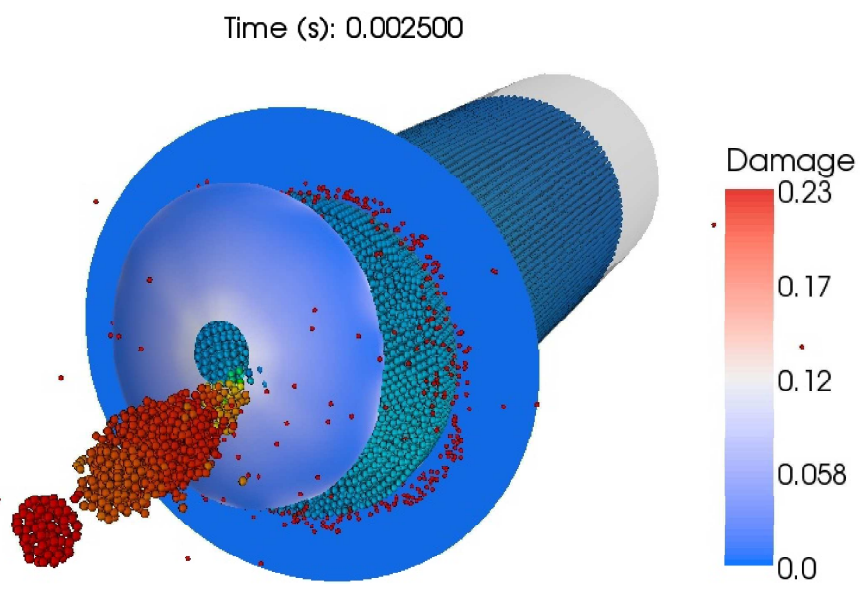

Figure 36: The simulation of test TF14A5 at $t=2.5 \mathrm{~ms}$

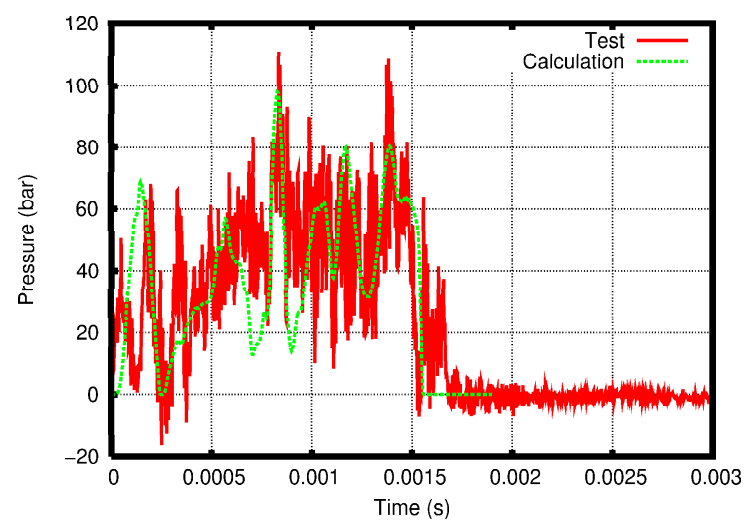

Figure 37: The pressure in the cylinder (test TF14A5) 


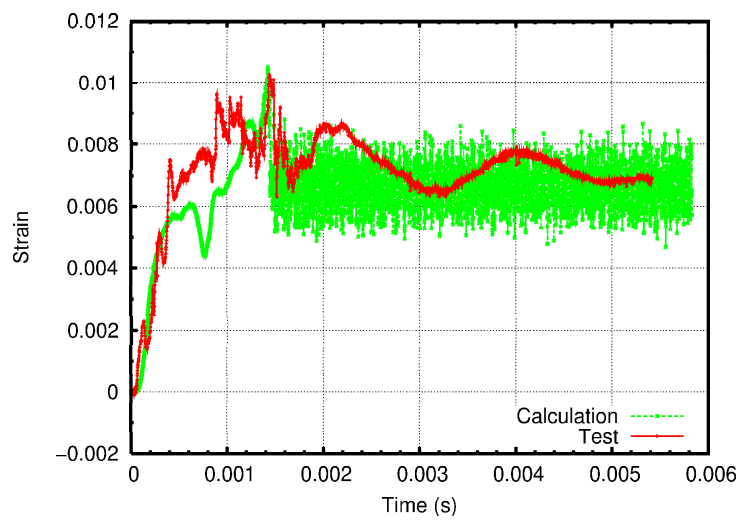

Figure 38: The deflection of the specimen (test TF14A5)

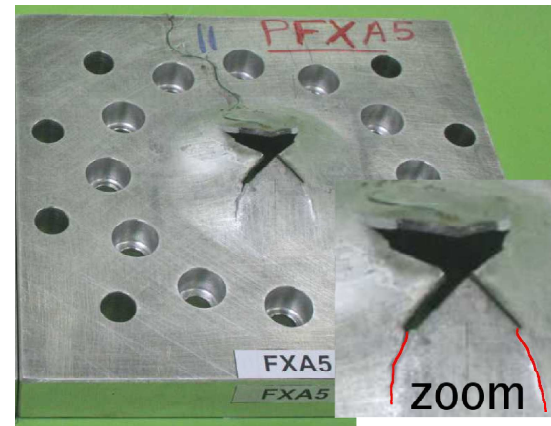

Figure 39: Propagation of the cracks (test FXA5)

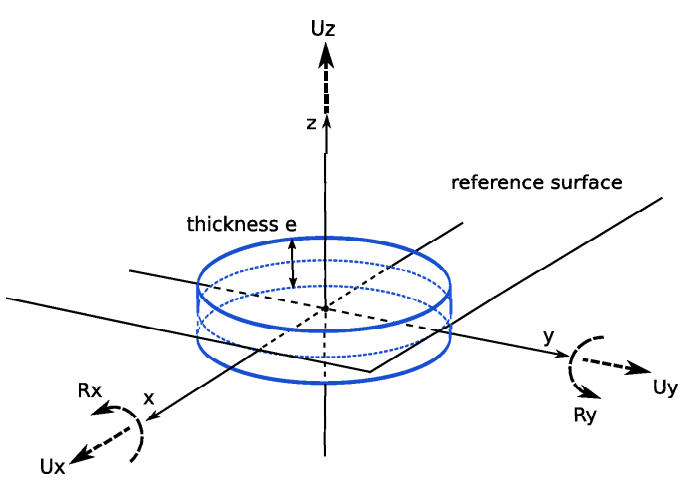

FIGURE 40: An SPHS particle 


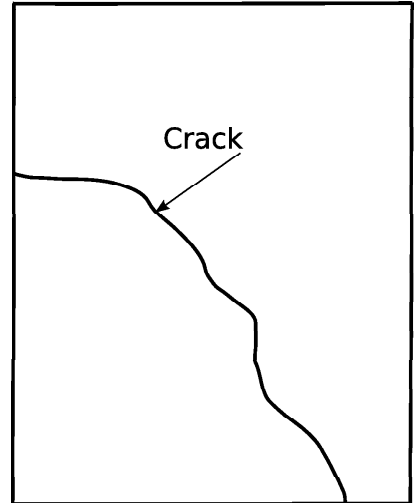

(a) Crack

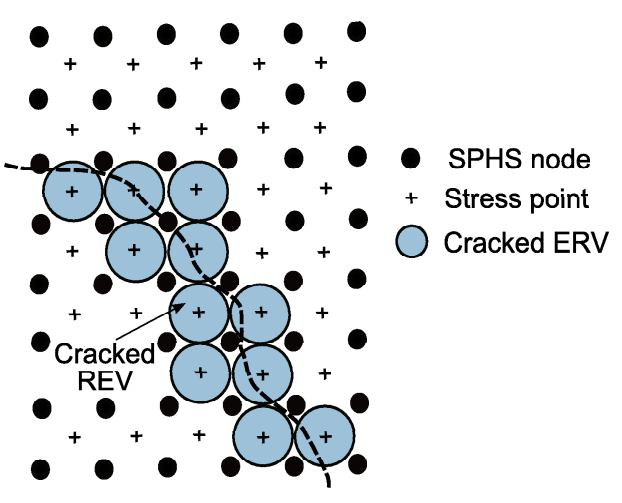

(b) The SPHS model

FiguRE 41: Representation of a crack using the SPHS model : example of a regular mesh of quadrilaterals



Figure 42: The mesh of the specimen (test FXA5) 


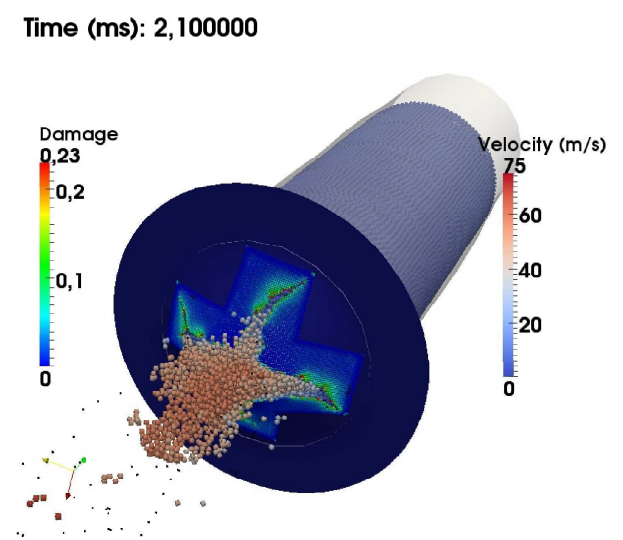

Figure 43: The simulation of test FXA5 at $t=2.1 \mathrm{~ms}$

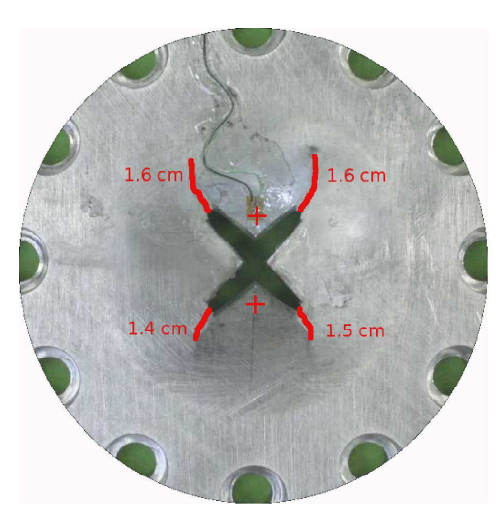

(a) Test

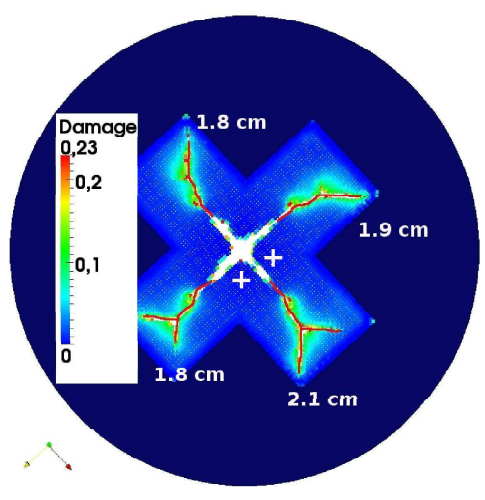

(b) Simulation

Figure 44: Comparison of the paths and lengths of the cracks (test FXA5) 


\begin{tabular}{lc}
\hline Test & Frequency $(H z)$ \\
\hline $1-9$ & $\sim 1,600$ \\
10 & $\sim 1,000-1,200$ \\
$12-13$ & 1,300 \\
\hline
\end{tabular}

TABLE 5: The frequencies observed experimentally

\begin{tabular}{ccccc}
\hline \multirow{2}{*}{ Test } & \multicolumn{4}{c}{ Characteristics of the specimen } \\
\cline { 2 - 5 } number & specimen & $e_{p}(\mathrm{~mm})$ & Precrack & $V(\mathrm{~m} / \mathrm{s})$ \\
\hline $1-7$ & E14A-B-C2 \& E14A-B-C-D5 & 25 & hole $(\mathrm{d}=14 \mathrm{~mm})$ & $2 \& 5$ \\
$8-9$ & E20A2 \& E20A5 & 25 & hole $(\mathrm{d}=20 \mathrm{~mm})$ & $2 \& 5$ \\
$10-11$ & F14A2 \& F14A2-5 & 2 & hole $(\mathrm{d}=14 \mathrm{~mm})$ & $2 \& 5$ \\
$12-13$ & FUA2 \& FUA5 & 2 & U-shaped precrack & $2 \& 5$ \\
$14-15$ & FXA2 \& FXA5 & 2 & X-shaped precrack & $2 \& 5$ \\
16 & TF14A5 & 1 & hole $(\mathrm{d}=14 \mathrm{~mm})$ & 5 \\
17 & TFA5 & 1 & no hole & 5 \\
18 & F14E3A5 & 2 & hole $(\mathrm{d}=14 \mathrm{~mm})+$ & 5 \\
19 & & 2 & precracks $(3 \mathrm{~mm})$ & \\
\hline
\end{tabular}

TABLE 4: Nomenclature and characteristics of the tests

$a$. The same specimen was used for tests 10 and 11 . 


\begin{tabular}{ccccc}
\hline$\epsilon_{p}^{s}$ & $\epsilon_{p}^{c}$ & $D_{c}$ & $\tau_{c}(\mu \mathrm{sec})$ & $\mathrm{a}$ \\
\hline 0.03 & 0.25 & 0.23 & 0.1 & 2 \\
\hline
\end{tabular}

TABLE 6: The material parameters of the damage law for AU4G 2024 (Suffis, 2004) 